\title{
Input to State Stability and Allied System Properties
}

\author{
S. N. Dashkovskiy, ${ }^{*}$ D. V. Efimov, ${ }^{* *}$ and E. D. Sontag ${ }^{* * *}$ \\ * University of Applied Sciences, Erfurt, Germany \\ ${ }^{* *}$ Universite de Bordeaux, Bordeaux, France \\ *** Rutgers University, New Jersey, USA
}

Received March 16, 2011

\begin{abstract}
The main results obtained in the field of input-state stable systems and systems with other similar characteristics that were published over the last two decades were reviewed.
\end{abstract}

DOI: $10.1134 /$ S0005117911080017

\section{INTRODUCTION}

The problems of design and analysis of the nonlinear control systems are at the center of attention of the scientific community for several decades, but it was only during the last two decades that an appreciable progress was marked in the design and application of various methods of the theory of nonlinear control. On the one hand, this success is due to the development of the computer engineering enabling fast and thorough study of the nonlinear processes, and to the increased demands on the control performance that can be satisfied by considering more complicated nonlinear models of the control plants and more complicated control algorithms. On the other hand, this is due to the development of an adequate theoretical apparatus for design and analysis of the nonlinear systems. The 1990s were noted for the advent and development of a whole series of nonlinear system methods that were christened "constructive" in [1]. The concept of stability of the dynamic systems from input to the state vector that is considered in what follows may be sorted with such "discoveries" $[2,3]$. It is distinguished for that it enables one to formalize the conditions for stability of the nonlinear system under input perturbations or, stated differently, to formalize the notion of system "robustness" to external perturbations.

The present review discusses the main results related with this concept and the allied notions of stability. The basic property of robust stability is introduced in Section 2. Sections 3 and 4 introduce various adjacent notions, and their use is considered in Section 5 . The auxiliary material and all abbreviations used in the review are compiled in Section 6, and the results left outside the present review are compiled in Section 7.

\section{CONCEPT OF ROBUST STABILITY}

It is common knowledge that for the linear stationary system

$$
\dot{x}=A x+B u,
$$

where $x \in \mathbb{R}^{n}$ and $u \in \mathbb{R}^{m}$ are the vectors of state and input, the Hurwitz stability of the matrix $A$ (all matrix eigenvalues have negative real part) implies global asymptotic stability for $u=0$. For an arbitrary (measurable) input $u$ and the initial conditions $x_{0} \in \mathbb{R}^{n}$, the system solution is set down as

$$
x(t)=e^{A t} x_{0}+\int_{0}^{t} e^{A(t-\tau)} B u(\tau) d \tau, \quad t \geqslant 0 .
$$


In this case, there exist constants $a>0$ and $b>0$ such that $\left|e^{A t}\right| \leqslant a e^{-b t}, t \geqslant 0$, and the system solutions satisfy the upper bound

$$
|x(t)| \leqslant a e^{-b t}\left|x_{0}\right|+a \int_{0}^{t} e^{-b(t-\tau)}|B||u(\tau)| d \tau \leqslant a e^{-b t}\left|x_{0}\right|+c \sup _{t \geqslant 0}|u(t)|,
$$

where $c=a|B| / b$. It necessarily follows from the last estimate for any $|u(t)| \rightarrow 0$ for $t \rightarrow \infty$ that $|x(t)| \rightarrow 0$ for $t \rightarrow \infty$, and boundedness of the input $u$ implies boundedness of the state vector $x(t)$. The estimate obtained coincides with the formulation of stability of the input-output operator of the linear system from the space $L_{\infty}$ to $L_{\infty}$ [4]. Other stability criteria that are similar to " $L_{\infty}$ to $L_{\infty} "$ are formulated in terms of the input-output operators as follows:

$$
\begin{array}{ll}
\text { " } L_{2} \text { in } L_{\infty} ": \quad & |x(t)| \leqslant a e^{-b t}\left|x_{0}\right|+c \int_{0}^{t}|u(\tau)| d \tau, \\
\text { " } L_{2} \text { in } L_{2} ": \quad & \int_{0}^{t}|x(\tau)| d \tau \leqslant a\left|x_{0}\right|+c \int_{0}^{t}|u(\tau)| d \tau .
\end{array}
$$

The first property defines in this case existence of the input-output operator with finite $\mathrm{H}_{2}$ norm, and the second condition defines existence of the operator with finite $H_{\infty}$ norm (it is assumed that the input signal is bounded). For the linear system, existence of any of these estimates amounts to the satisfaction of the rest of them, that is, if a linear system is asymptotically stable at zero input, then it has bounded solutions for any bounded input. An intuitive extension of this property to the class of nonlinear stationary systems proves to be invalid. To illustrate this fact, we consider the following example [5]:

$$
\dot{x}=-3 x+\left(1+2 x^{2}\right) u .
$$

This system is globally asymptotically stable for zero input, but for the initial conditions $x_{0}=2$ and $u(t)=1$ the corresponding solution $x(t)=\left(3-e^{t}\right) /\left(3-2 e^{t}\right)$ goes to infinity in a finite time! Moreover, another example: for any constant input $u(t) \geqslant 2$, the system

$$
\dot{x}=-x+x u
$$

is unstable, but it is possible to demonstrate [6] that for the asymptotically decaying input its solutions are defined for all $t \geqslant 0$ and tend asymptotically to zero (it suffices to consider for that the Lyapunov function $V(x)=\frac{1}{2} x^{2}$ ), that is, the system is stable in the sense of " $L_{2}$ to $L_{\infty}$ " but does not have stability " $L_{\infty}$ to $L_{\infty}$." Therefore, it turns out that for the nonlinear systems the property of global stability to the external bounded and/or decaying perturbations does not follow from global (exponential) stability in the absence of inputs. The theory of input-state stable systems whose basic premises are presented below answers the question as to when the nonlinear system features robustness to the external perturbations.

\subsection{Formulation of the Problem}

We consider nonlinear dynamic systems with model representable as

$$
\dot{x}=f(x, u)
$$

or

$$
\dot{x}=f(x)+G(x) u,
$$


where $x \in \mathbb{R}^{n}$ is the state vector of systems (2.3) and (2.4) and $u \in \mathbb{R}^{m}$ is the input vector. In some cases, we consider such systems with the output $y=h(x)$, where $h: \mathbb{R}^{n} \rightarrow \mathbb{R}^{p}$. Let the function $f: \mathbb{R}^{n} \times \mathbb{R}^{m} \rightarrow \mathbb{R}^{n}$ in (2.3) and, correspondingly, the functions $f: \mathbb{R}^{n} \rightarrow \mathbb{R}^{n}$ and $G: \mathbb{R}^{n} \rightarrow \mathbb{R}^{n \times m}$ in (2.4) be continuous and locally Lipschitzian (continuously differentiable), $f(0,0)=0$ and, correspondingly, $f(0)=0$. We assume that the control or input of systems (2.3) and (2.4) is a measurable and locally bounded almost everywhere function $u: \mathbb{R}_{+} \rightarrow \mathbb{R}^{m}$ (for more detailed definition of function measurability see, for example, [7]). The set of all such functions satisfying the condition

$$
\|u\|=\operatorname{ess} \sup \{|u(t)|, \quad t \geqslant 0\}<+\infty
$$

is denoted by $M_{\mathbb{R}^{m}}$ (the main definitions and notations used in the present paper, as well as some basic properties of systems (2.3) and (2.4) are compiled in Section 6). The symbol $x\left(t, x_{0}, u\right)$ denotes the trajectory of system (2.3) for $t \geqslant 0$ with the initial conditions $x_{0} \in \mathbb{R}^{n}$ and $u \in M_{\mathbb{R}^{m}}$ (sometimes, if the arguments are understandable from the context, we use $x(t)$ for $x\left(t, x_{0}, u\right)$ ). It is required to define the robust stability of these systems to inputs, work out the necessary and sufficient stability conditions, characterize this property in terms of the Lyapunov functions, and establish the relation between the properties of globally asymptotic and robust stability. System (2.3) generalizes (2.4). Therefore, below all definitions are considered for system (2.3), and special references to (2.4) are made wherever necessary. Some notations and standard stability definitions of systems (2.3) and (2.4) are given in Section 6.

\subsection{Stability from Input to State Vector}

More than two decades ago one of the present authors suggested [8] a definition of robust stability of system (2.3) formulated by generalizing the estimate established for the stable linear systems (the definitions of functions from the classes $\mathcal{K} \mathcal{L}$ and $\mathcal{K}$ that are used below are given in Section 6).

Definition 2.1 [8]. System (2.3) is called input-to-state stable if for any input $u \in M_{\mathbb{R}^{m}}$ and $x_{0} \in \mathbb{R}^{n}$ there exist functions $\beta \in \mathcal{K} \mathcal{L}$ and $\gamma \in \mathcal{K}$ such that

$$
\left|x\left(t, x_{0}, u\right)\right| \leqslant \beta\left(\left|x_{0}\right|, t\right)+\gamma(|| u||)
$$

for $\forall t \geqslant 0$. The function $\gamma$ is called the nonlinear gain.

We abbreviate this new property of system (2.3) by ISS. Since $\max \{a, b\} \leqslant a+b \leqslant 2 \max \{a, b\}$ is satisfied for any $a, b \in \mathbb{R}_{+}$, there exists an equivalent formulation of ISS:

$$
\left|x\left(t, x_{0}, u\right)\right| \leqslant \max \left\{\beta\left(\left|x_{0}\right|, t\right), \gamma(|| u||)\right\}
$$

for all $x_{0} \in \mathbb{R}^{n}, u \in M_{\mathbb{R}^{m}}$ and some $\beta \in \mathcal{K} \mathcal{L}, \gamma \in \mathcal{K}$. For the solutions of the linear system (2.1), the determined estimate (2.2) satisfies, obviously, the definition of ISS for $\beta(s, t)=a s e^{-b t}$ and $\gamma(s)=c s$. The functions of classes $\mathcal{K} \mathcal{L}$ and $\mathcal{K}$ allow one to denote briefly in the general form the estimates for solutions of the nonlinear systems as it is done with the use of the linear gains and exponential estimates in the linear systems. We notice that it is advisable to determine more precise estimates of the solutions for determination of ISS for applications. Depending on the system, it may be more convenient to use for that (2.6) instead of (2.5). There exists a more general form of this defining inequality [9] which is especially useful at considering connections of the ISS systems where signals of more than one system are input in a single system. Now, we consider an extended description of the property of ISS and equivalent means for defining it for which purpose we introduce some accompanying properties of the dynamic systems.

Definition 2.2 [10]. System (2.3)

(a) is called 0-locally stable (0-LS) if at the point $x_{0}=0$ for $u=0$ the map $x_{0} \rightarrow x$ is continuous (local Lyapunov stability at the origin); 
(b) has asymptotic gain if for all $u \in M_{\mathbb{R}^{m}}$ and $x_{0} \in \mathbb{R}^{n}$ there exists function $\gamma \in \mathcal{K}$, the nonlinear asymptotic coefficient of the system, such that

$$
\varlimsup_{t \rightarrow+\infty}\left|x\left(t, x_{0}, u\right)\right| \leqslant \gamma(\|u\|)
$$

(c) has uniform asymptotic gain if the upper limit in the last inequality exists uniformly in $x_{0}$ and $u$, that is, there exists a function $\gamma \in \mathcal{K}$ such that $\forall \varepsilon>0, \forall \kappa>0 \exists T(\varepsilon, \kappa)>0$ :

$$
\left|x_{0}\right| \leqslant \kappa \Rightarrow \sup _{t \geqslant T(\varepsilon, \kappa)}\left|x\left(t, x_{0}, u\right)\right| \leqslant \gamma(\|u\|)+\varepsilon
$$

(d) has the limit property if for all $u \in M_{\mathbb{R}^{m}}$ and $x_{0} \in \mathbb{R}^{n}$ there exists a function $\rho \in \mathcal{K}$ such that

$$
\inf _{t \geqslant 0}\left|x\left(t, x_{0}, u\right)\right| \leqslant \rho(\|u\|) .
$$

Condition (b) of the last definition may be interpreted as the "limit boundedness" uniform in the initial conditions for all solutions of the ISS system. This condition may also be interpreted as an extension of the system attractivity condition to the case of $u \neq 0$. Condition (d) implies that for any initial conditions $x_{0} \in \mathbb{R}^{n}$ and $u \in M_{\mathbb{R}^{m}}$ their corresponding trajectory of system $x\left(t, x_{0}, u\right)$ enters the neighborhood $\rho(\|u\|)$ of the origin but does not necessarily remain there (according to the limit property, then this trajectory may be unbounded).

Definition 2.3 [11]. System (2.3) is referred to as robust stable if there exists function $\rho \in \mathcal{K}_{\infty}$ such that from any permissible, possibly nonstationary, law of feedback control

$$
u=k(t, x),
$$

which uniformly globally asymptotically stabilizes system (2.3) (uniformly in $k$ ) it follows that the condition

$$
|k(t, x)| \leqslant \rho(|x|) \quad \forall x \in \mathbb{R}^{n} \text { and almost all } t \geqslant 0
$$

is satisfied.

By the permissible feedback control law is meant the law $k(t, x)$ such that for $x(0) \in \mathbb{R}^{n}$ the system solution

$$
\dot{x}=f(x, k(t, x))
$$

exists and is defined at least for small $t$, and two such solutions coincide on their interval of existence. The function $k(t, x)$ also may represent both the unaccounted dynamics or external perturbation $k(t)$. In this case, $\rho$ is called the stability margin, and, according to the definition, if the external perturbation $k$ is bounded in amplitude by the stability margin $\rho$, then the system retains stability (is asymptotically stable, robust to perturbations bounded by $\rho$ ). Importance and relation with ISS of the properties introduced in Definitions 2.2 and 2.3 are explained by the following theorem.

Theorem 2.1 [10, 12]. The following properties are equivalent for system (2.3):

- ISS;

- existence of uniform asymptotic gain;

- $0-L S$ and existence of asymptotic gain;

- 0-LS and existence of the limit property;

- robust stability. 
Therefore, the property of ISS may be introduced directly through the notions of robust stability or asymptotic gain in input. These equivalent definitions give a better insight into the features of ISS that are hidden in estimate (2.5) from Definition 2.1. We notice one more distinction of ISS, its invariance to the transformation of coordinates. If $x$ is the state vector of system ISS, then by introducing a homeomorphic transformation to new coordinates $\widehat{x}=S(x)$, where $S$ is continuous and exists continuously $S^{-1}, S(0)=0$ ), we get that in the new coordinates $\widehat{x}$ the system is also ISS with the modified functions $\beta$ and $\gamma$. We emphasize that not any property of stability is independent of the nonlinear transformation of coordinates. For example, the property of exponential stability is not such [2]! ISS and other notions may be readily generalized to other types of systems such as the discrete, hybrid, or delay systems, as well as more general classes of systems [13-15].

\subsection{ISS of Lyapunov Function}

The apparatus of the Lyapunov functions [16] is traditionally used to analyze autonomous systems for stability (for $u=0$ ). This property of stability presumes availability of system input. The fundamental achievement of the direct Lyapunov method is the possibility to analyze (asymptotic) behavior of the system solutions without calculating the solutions proper as time function and the initial conditions which is a hard problem for the nonlinear systems. The inequalities for some functions of the state vector and their derivatives are checked by substituting the functions of the right side of the system. This approached first was extended to the input systems in the theory of dissipative systems (see Definition 6.3 of this kind of stability in Section 6, [17, 18]) where the system solutions were estimated using the inequalities depending on the system input, output, and state for the derivatives of the corresponding positive definite functions (supply rates). It turns out that such inequalities also enable one to determine the qualitative and quantitative characteristics of behavior of the solutions of the perturbed systems without calculating the solutions proper in the time domain. A.M. Lyapunov understood and introduced his functions namely with this purpose in view. In this connection the apparatus discussed below was named the Lyapunov functions.

Definition 2.4 [8]. The smooth function $V: \mathbb{R}^{n} \rightarrow \mathbb{R}_{+}$is called the ISS Lyapunov function if for it there are the functions $\alpha_{1}, \alpha_{2} \in \mathcal{K}_{\infty}, \alpha_{3}, \chi \in \mathcal{K}$ such that the conditions

$$
\begin{gathered}
\alpha_{1}(|x|) \leqslant V(x) \leqslant \alpha_{2}(|x|) \quad \forall x \in \mathbb{R}^{n}, \\
|x| \geqslant \chi(|u|) \Rightarrow L_{f(x, u)} V(x) \leqslant-\alpha_{3}(|x|) \quad \forall x \in \mathbb{R}^{n}, u \in \mathbb{R}^{m},
\end{gathered}
$$

are satisfied.

We notice that generally speaking the gain $\chi$ is other than the gain $\gamma$ introduced in the definition of ISS. The notion of input to state (ISDS) dynamic stability equivalent to ISS was introduced in [19]. However, it disregards the effect of decay of the influence of earlier values of the input on the current values of the state vector. Additionally, the definition of ISDS and the corresponding ISDS Lyapunov function are introduced so that the corresponding gains coincide. An equivalent definition of the ISS Lyapunov function is obtained if condition (2.7) is replaced by

$$
D V(x) f(x, u) \leqslant \theta(|u|)-\alpha(|x|) \quad \forall x \in \mathbb{R}^{n}, u \in \mathbb{R}^{m},
$$

where $\alpha, \theta \in \mathcal{K}_{\infty}$.

Theorem 2.2 [8, 12]. System (2.3) is ISS if and only if there exists for it an ISS Lyapunov function.

We notice that for $u=0$ the ISS Lyapunov function is reduced to the standard Lyapunov function for the system asymptotic stability. It was demonstrated in [20] that if a system has the 
Lyapunov function, then it has also a smooth "exponential" Lyapunov function $V: \mathbb{R}^{n} \rightarrow \mathbb{R}_{+}$such that

$$
\alpha_{1}(|x|) \leqslant V(x) \leqslant \alpha_{2}(|x|), \quad D V(x) f(x, u) \leqslant \theta(|u|)-V(x)
$$

is satisfied for all $x \in \mathbb{R}^{n}, u \in \mathbb{R}^{m}$, and some $\alpha_{1}, \alpha_{2} \in \mathcal{K}_{\infty}, \theta \in \mathcal{K}$.

An important useful mechanism to handle the ISS Lyapunov functions enabling one to modify the functions $\alpha, \alpha_{3}, \chi, \theta$ at zero or on infinity was proposed in [21]. By the definition of the dissipative systems (Definition 6.3) and form of the ISS Lyapunov function, the ISS systems make up a subclass of dissipative systems ( $V$ is the supply rate, $(\theta(|u|)-\alpha(|x|))$ is the expenditure function). Let us consider the simplest examples illustrating the relation between the method of Lyapunov functions and the property of ISS. First we notice that the linear system (2.1) is ISS if and only if $A$ is a Hurwitz matrix and $V(x)=x^{\mathrm{T}} P x$, where $P=P^{\mathrm{T}}>0$ is the solution of the corresponding Lyapunov equation $A^{\mathrm{T}} P+P A<0$, can be used as the Lyapunov function. This follows from the above reasoning for the linear systems. Now we consider the nonlinear system

$$
\dot{x}=-x^{3}+u, \quad x, u \in \mathbb{R} .
$$

By taking $V(x)=\frac{1}{2} x^{2}, \alpha_{3}(s)=\frac{1}{2} s^{4}, \chi(s)=\left(\frac{1}{\sqrt{2}} s\right)^{\frac{2}{3}}, s \geqslant 0$, we obtain that

$$
\dot{V}=x\left(-x^{3}+u\right)<-\frac{1}{2} x^{4}=-\alpha_{3}(|x|)
$$

if $V(x)>\chi(|u|)$. This means that this system is ISS, and $V(x)=\frac{1}{2} x^{2}$ can be used as its ISS Lyapunov function.

\subsection{ISS Stabilizability}

We present the results enabling one to endow the given system of form (2.3) with the ISS property and introduce the following definition.

Definition 2.5 [11]. System (2.3) is referred to as stabilizable if there exists for it a control law

$$
u=k(x),
$$

where $k$ is continuous and differentiable function, such that the closed-loop control system is globally asymptotically stable at the origin of the state space. In what follows, we call (2.3) the ISS stabilizable system if for it there exists a control law

$$
u=k(x)+v,
$$

where $v$ is the new input vector rendering to the closed-loop system the property of ISS in input $v$. Finally, we call (2.3) weakly ISS stabilizable if for it there exists a control law

$$
u=k(x)+\Omega(x) v
$$

where the matrix function $\Omega$ is continuous, differentiable, and invertible for each $x$, such that the closed-loop system is ISS in input $v$.

Obviously, the ISS stabilizable system is weakly ISS stabilizable for $\Omega(x)=I$. Additionally, by definition of ISS, the ISS stabilizable and weakly ISS stabilizable systems are simultaneously stabilizable because for $v=0$ we obtain a globally asymptotically stable system. Moreover, the following results are valid. 
Theorem 2.3 [11]. If system (2.3) is stabilizable, then it is also weakly ISS stabilizable,

Theorem 2.4 [8]. If system (2.4) is stabilizable, the it is also ISS stabilizable.

It was also proved in [22] that if (2.4) is a globally and asymptotically controllable system, that is, for any $x_{0} \in \mathbb{R}^{n}$ there exists $u \in M_{\mathbb{R}^{m}},\|u\| \leqslant \sigma\left(\left|x_{0}\right|\right), \sigma \in \mathcal{K}$, such that $\left|x\left(t, x_{0}, u\right)\right| \leqslant \beta\left(\left|x_{0}\right|, t\right)$, $\beta \in \mathcal{K} \mathcal{L}, t \geqslant 0)$, then there exists a feedback control law $u=k(x)+v$, possibly with a discontinuous function $k$, such that the closed-loop system is ISS. Since system (2.4) with the control $k$ is generally discontinuous, a special determination of the solutions of such perturbed system is used in [22]. These results establish the conditions for principal solvability of the problem of ISS stabilization without offering a choice of the functions $k$ and $\Omega$. Particular methods of designing the robust stabilizing control laws for various classes of system will be considered in Section 5 .

\subsection{Relation between the Input-State and ISS Operators}

Let us consider some other properties of the ISS system which rely on studying the operators of mapping the space of inputs onto that of states of the nonlinear dynamic system which are similar to those considered in the Introduction for the linear system.

Definition 2.6 [6]. System (2.3) will be said to have the property " $L^{\infty}$ to $L^{\infty}$ " if there exist functions $\alpha, \gamma \in \mathcal{K}_{\infty}$ and $\beta \in \mathcal{K} \mathcal{L}$ such that

$$
\alpha\left(\left|x\left(x_{0}, t, u\right)\right|\right) \leqslant \beta\left(\left|x_{0}\right|, t\right)+\sup _{s \in[0, t]} \gamma(|u(s)|) \quad \forall t \geqslant 0
$$

is satisfied $\forall x_{0} \in \mathbb{R}^{n}, \forall u \in M_{\mathbb{R}^{m}}$.

Definition 2.7 [6]. System (2.3) will be said to have the property " $L^{2}$ to $L^{\infty}$ " if there exist functions $\alpha, \gamma \in \mathcal{K}_{\infty}$ and $\beta \in \mathcal{K} \mathcal{L}$ such that

$$
\alpha\left(\left|x\left(x_{0}, t, u\right)\right|\right) \leqslant \beta\left(\left|x_{0}\right|, t\right)+\int_{0}^{t} \gamma(|u(s)|) d s \quad \forall t \geqslant 0
$$

is satisfied $\forall x_{0} \in \mathbb{R}^{n}, \forall u \in M_{\mathbb{R}^{m}}$.

Definition 2.8 [6]. System $(2.3)$ will be said to have the property " $L^{2}$ to $L^{2}$ " if there exist functions $\alpha, \gamma, \kappa \in \mathcal{K}_{\infty}$ such that

$$
\int_{0}^{t} \alpha(|x(s)|) d s \leqslant \kappa\left(\left|x_{0}\right|\right)+\int_{0}^{t} \gamma(|u(s)|) d s \quad \forall t \geqslant 0
$$

is satisfied $\forall x_{0} \in \mathbb{R}^{n}, \forall u \in M_{\mathbb{R}^{m}}$.

Definition 2.6 has a natural continuation on the nonlinear systems of the notion of the finite $L_{1}$ operator from input to output under the conditions of nonlinear change of coordinates [6]. Definition 2.7 implies that in this case there exists an input-output operator with a finite $\mathrm{H}_{2}$ norm. Definition 2.8 extends to the nonlinear systems the notion of the operator with finite $H_{\infty}$ norm. The following lemma is true.

Lemma 2.1 [6]. System (2.3) is ISS if and only if it has any of the properties given in Definitions 2.6 and 2.8.

This conclusion established one more parallel between the properties of ISS for the nonlinear system and the globally asymptotic stability of the linear where this relation is satisfied as well. The property given in Definition 2.7 is weaker than ISS. We notice that a dynamic system of such type 
features extendability of solutions. This property of the dynamic system (2.3) was named in [6] the integral stability from input to the state vector (iISS). We notice an obvious relation of the properties of ISS and iISS of the nonlinear dynamic systems [6]: if system (2.3) is ISS, then it is iISS as well. The property of iISS is the first allied ISS property mentioned in this review which is based on the "ISS concept," that is, as will be shown below, it has similar ways of formulation, relation with stability of the linear systems, and equivalent expression in terms of the Lyapunov function. The features of the iISS property, as well as other additional properties of stability obtained within the framework of the ISS concept are discussed in Section 3.

\section{CONCOMITANT ISS PROPERTIES}

Let us consider the iISS property and other characteristics of the dynamic systems formulated on the basis of the ISS concept.

\subsection{Integral Stability from Input to State Vector}

We stress an important feature of the " $L^{2}$ to $L^{\infty}$ " stable systems.

Lemma 3.1 [6]. If the solutions of system (2.3) satisfy the estimate from Definition 2.7, then for any $u$ such that

$$
\int_{0}^{\infty} \gamma(|u(s)|) d s<\infty,
$$

and any $x_{0} \in \mathbb{R}^{n}$ it follows that $x\left(t, x_{0}, u\right) \rightarrow 0$ for $t \rightarrow+\infty$.

Now we present the necessary and sufficient conditions for the iISS property of the dynamic system for which purpose we introduce several useful definitions.

Definition 3.1 [23]. The continuously differentiable function $V: \mathbb{R}^{n} \rightarrow \mathbb{R}_{+}$is called the iISS Lyapunov function if for it there exist functions $\alpha_{1}, \alpha_{2} \in \mathcal{K}_{\infty}, \sigma \in \mathcal{K}$ and positive definite continuous function $\alpha_{3}$ such that

$$
\alpha_{1}(|x|) \leqslant V(x) \leqslant \alpha_{2}(|x|) \quad \forall x \in \mathbb{R}^{n}
$$

and

$$
D V(x) f(x, u) \leqslant-\alpha_{3}(|x|)+\sigma(|u|) \quad \forall x \in \mathbb{R}^{n}, u \in \mathbb{R}^{m} .
$$

We emphasize the main distinction between the ISS and iISS Lyapunov functions: for the ISS system the function $\alpha_{3} \in \mathcal{K}_{\infty}$. Now we consider system (2.3) with output

$$
\dot{x}=f(x, u), \quad y=h(x),
$$

where $h: \mathbb{R}^{n} \rightarrow \mathbb{R}^{p}$ is a continuous function. For any $x_{0} \in \mathbb{R}^{n}$ and $u \in M_{\mathbb{R}^{m}}$, we denote $y\left(t, x_{0}, u\right)=$ $h\left(x\left(t, x_{0}, u\right)\right)$ for all $t \geqslant 0$, where the solution of system (3.1) is determined. Below we denote by $Z=\left\{x \in \mathbb{R}^{n}: h(x)=0\right\}$ the set of zero-dynamics of system (3.1).

Definition $3.2[23,24]$. System (3.1) is referred to as detectable in zero in output $h$ if

$$
y\left(t, x_{0}, 0\right)=0, \quad t \geqslant 0 \Rightarrow \lim _{t \rightarrow+\infty}\left|x\left(t, x_{0}, 0\right)\right|=0
$$

is satisfied for any $x_{0} \in \mathbb{R}^{n}$. System (3.1) is referred to as $h$-dissipative if there exist continuously differentiable, proper unbounded and positive definite function $W: \mathbb{R}^{n} \rightarrow \mathbb{R}_{+}, \sigma \in \mathcal{K}$ and positive definite function $\alpha_{4}$ such that

$$
D W(x) f(x, u) \leqslant-\alpha_{4}(|h(x)|)+\sigma(|u|) \quad \forall x \in \mathbb{R}^{n}, u \in \mathbb{R}^{m} .
$$


If this property is true for the smooth function $W$, then system (3.1) is referred to as smoothly $h$-dissipative.

For a system detected at zero in output $h$, the set $Z$ is invariant for $u=0$ and formed by the solutions of system (3.1) with input and output identically zero (solutions of the zero-dynamics system) tending asymptotically to the equilibrium at the origin.

Theorem 3.1 [23, 25]. The following statements are equivalent for system (2.3):

(1) system is iISS;

(2) system has a smooth iISS Lyapunov function;

(3) there exists an output $h$ making the system smoothly h-dissipative and detectable at zero;

(4) system is globally asymptotically stable for $u=0$, and there exists a smooth positive definite and proper unbounded function $V: \mathbb{R}^{n} \rightarrow \mathbb{R}_{+}$such that

$$
D V(x) f(x, u) \leqslant \sigma(|u|)
$$

for all $x \in \mathbb{R}^{n}, u \in \mathbb{R}^{m}$ and some $\sigma \in \mathcal{K}$;

(5) there exists a smooth positive definite and proper unbounded function $W: \mathbb{R}^{n} \rightarrow \mathbb{R}_{+}$, functions $\gamma, \rho \in \mathcal{K}_{\infty}$, and positive definite function $b$ with $\int_{0}^{+\infty} \frac{d r}{1+b(r)}=+\infty$ such that

$$
|x| \geqslant \rho(|u|) \Rightarrow D W(x) f(x, u)<\gamma(|u|) b[W(x)]
$$

is satisfied for all $x \in \mathbb{R}^{n} \backslash\{0\}, u \in \mathbb{R}^{m}$;

(6) there exist functions $W, \gamma, \rho, b$ satisfying conditions of item (5) and positive definite function $\alpha$ such that

$$
|x| \geqslant \rho(|u|) \Rightarrow D W(x) f(x, u) \leqslant-\alpha(|x|)+\gamma(\mid u) b[W(x)]
$$

is satisfied for all $x \in \mathbb{R}^{n} \backslash\{0\}, u \in \mathbb{R}^{m}$.

Therefore, the property of iISS has the same wide set of equivalent means of definition as ISS, the difference being only in robustness of the system to various input actions. The general style of describing these properties allows one to classify them with the same concept of stability. We notice that it was proved in [26] that iISS and weakened property " $L^{2}$ to $L^{2}$ " are equivalent. Here, for all $x_{0} \in \mathbb{R}^{n}, u \in M_{\mathbb{R}^{m}}$ there are $\alpha, \gamma, \kappa, \chi \in \mathcal{K}_{\infty}$ such that

$$
\int_{0}^{t} \alpha(|x(s)|) d s \leqslant \kappa\left(\left|x_{0}\right|\right)+\chi\left(\int_{0}^{t} \gamma(|u(s)|) d s\right) \quad \forall t \geqslant 0
$$

is satisfied.

A distinction of this property from that introduced in Definition 2.8 lies in the function $\chi$ whose occurrence may be interpreted as " $L^{p}$ to $L^{q}$ " stability of the system for $q \neq p$. The following system is considered as an example of the iISS system which is not ISS:

$$
\dot{x}=-\frac{x}{x^{2}+1}+u, \quad x, u \in \mathbb{R} .
$$

Obviously, this system is not ISS. For example, $\dot{x} \geqslant \frac{1}{2}$ is satisfied for $u \equiv 1$ and the solution will be unbounded for any initial condition. Let us consider the Lyapunov functions $V(x)=|x|$ as 
candidates for iISS. This function is differentiable over the entire numerical axis, excepts for the origin. By differentiating it, we obtain in virtue of the system equations that

$$
\dot{V}=-\frac{|x|}{x^{2}+1}+\operatorname{sgn}(x) u \leqslant-\frac{|x|}{x^{2}+1}+|u|, \quad x \neq 0 .
$$

Determination of $\alpha_{3}(|x|)=\frac{|x|}{x^{2}+1}$ and $\sigma(|u|)=|u|$ shows that the function $V$ is an iISS Lyapunov function for the system under consideration. The aforementioned system

$$
\dot{x}=-x+x u,
$$

can be shown to be an iISS system; moreover, any bilinear system

$$
\dot{x}=\left(A+\sum_{i=1}^{m} u_{i} A_{i}\right) x+B u, \quad x \in \mathbb{R}^{n}, \quad u=\left(u_{1}, \ldots, u_{m}\right)^{\mathrm{T}} \in \mathbb{R}^{m},
$$

is iISS if and only if $A, A_{1}, \ldots, A_{m}$ are Hurwitzian matrices [3].

\subsection{Local Stability from Input to State Vector}

An interesting generalization of ISS is represented by its local version (LISS) [10, 27, 28].

Definition 3.3. System (4.2) is referred to as locally stable input to state stable (LISS) if there exist positive numbers $\rho^{0}$ and $\rho^{u}$ and functions $\gamma \in \mathcal{K}_{\infty}$ and $\beta \in \mathcal{K} \mathcal{L}$ such that

$$
x\left(t, x_{0}, u\right)<\beta\left(\left|x_{0}\right|, t\right)+\gamma\left(\|u\|_{\infty}\right), \quad t \geqslant 0
$$

is satisfied for all initial values $\left|x^{0}\right| \leqslant \rho^{0}$ and inputs $\|u\| \leqslant \rho^{u}$.

Obviously, for $\rho^{0}=\infty, \rho^{u}=\infty$ we obtain the ordinary ISS property. We notice that the set of LISS systems includes the class of iISS as a subset which in turn includes the set of ISS systems. The Lyapunov function for the LISS systems is defined similar to the case of ISS. At that, the implication

$$
V(x) \geq \gamma(|u|) \Longrightarrow D V(x) f(x, u) \leq-\alpha(V(x))
$$

must be satisfied only for $|x| \leqslant \rho^{0}$ and $|u| \leqslant \rho^{u}$. The local versions of uniform asymptotic gain, asymptotic stability, and so on can be defined similarly. We consider the following system

$$
\dot{x}=-x+x^{2}+u, \quad x, u \in \mathbb{R}
$$

by way of example of the LISS system. For the zero input, this system has two equilibria and, therefore, is not globally asymptotically stable and ISS. However, if constraints are imposed on the initial conditions and the input, for example, by requiring that $|x(0)|<\frac{1}{2}$ and $|u|<\frac{1}{4}$, then one can readily make sure that this system is LISS for which purpose $V(x)=|x|$ can be used as the LISS of the Lyapunov function. We consider below systems with output and the corresponding properties of robust stability.

\subsection{Stability from Output and Input to State Vector}

Let us consider the dual ISS property relative to the output vector of the system

$$
\dot{x}=f(x), \quad y=h(x),
$$

where the function $f$ is locally Lipschitzian and continuous; $h$ is continuously differentiable, and $f(0)=0$. For the linear systems, such property was christened "detectability" or minimum phase property. 
Definition 3.4 [29]. System (3.4) is called output to state stable (OSS) if there exist functions $\beta \in \mathcal{K} \mathcal{L}$ and $\gamma \in \mathcal{K}$ such that

$$
\left|x\left(t, x_{0}\right)\right| \leqslant \beta\left(\left|x_{0}\right|, t\right)+\gamma\left(\| y_{[0, t]}||\right)
$$

is satisfied for all $x_{0} \in \mathbb{R}^{n}$ and all $t \in\left[0, t_{\max }\right)$. Here, $t_{\max }=t_{\max }\left(x_{0}\right) \leqslant+\infty$ defines the interval over which the solution of system (3.4) is defined a priori, $y(t)=h\left[x\left(t, x_{0}\right)\right]$ is the output function, and $y_{[0, t]}$ is a contraction of the solution $y(t)$ to the time interval $[0, t]$.

Definition 3.5 [29]. The function $V: \mathbb{R}^{n} \rightarrow \mathbb{R}_{+}$is called the OSS Lyapunov function for system (3.4) if:

(1) there exist functions $\alpha_{1}, \alpha_{2} \in \mathcal{K}_{\infty}$ such that

$$
\alpha_{1}(|x|) \leqslant V(x) \leqslant \alpha_{2}(|x|) \quad \forall x \in \mathbb{R}^{n}
$$

is satisfied;

(2) the function $V$ is continuously differentiable along the trajectories of system (3.4), at that, there are functions $\alpha, \sigma \in \mathcal{K}_{\infty}$ :

$$
D V(x) f(x) \leqslant-\alpha(|x|)+\sigma(|y|) \quad \forall x \in \mathbb{R}^{n} \quad \text { and } \quad t \in\left[0, t_{\max }\right) .
$$

The following theorem is valid.

Theorem 3.2 [29]. System (3.4) is OSS if and only if it has an OSS Lyapunov function.

Therefore, substitution of output for input in the estimates from Definitions 2.1-3.4 leads to a similar modification of the Lyapunov functions. We present another interesting result.

Lemma 3.2 [30]. Let the system (2.4) have globally definite normal form [31], that is, there be a smooth transform of coordinates $x=\Phi(y, z)$ such that in new coordinates Eqs. (2.4) are given by

$$
\begin{gathered}
\dot{z}=q(z, y), \quad z \in \mathbb{R}^{n-p} ; \\
\dot{y}=a(z, y)+B(z, y) u, \quad y \in \mathbb{R}^{p} .
\end{gathered}
$$

System (2.4) is OSS if and only if subsystem (3.5) (on the submanifold of zero dynamics) has the property of ISS relative to $y$.

We notice that the ISS property of the $z$-subsystem must be understood only for the output functions of system (2.4) and not for any measured input $y$ of $z$-subsystem. Determination of stability from output to state and input (in the case where the state and input vectors are bounded not only in the output norm, but also the norms of its derivatives) can be found in [32]. Such property can be regarded as a development of the OSS property and robust minimum phase [5]. Some variants of extension of the ISS property to the systems with output (3.1) were proposed in $[29,33]$. In particular, the following property is a roughened analog of the detectability in zero (see Definition 3.2 and [24]); it can also be regarded as an extension of the OSS property to the systems with input (3.1). In the case at hand, the vector function $h$ is continuously differentiable.

Definition 3.6 [29]. System (3.1) is called the input/output to state vector stable (IOSS) if there exist functions $\beta \in \mathcal{K} \mathcal{L}$ and $\gamma_{1}, \gamma_{2} \in \mathcal{K}$ such that

$$
\left|x\left(t, x_{0}\right)\right| \leqslant \beta\left(\left|x_{0}\right|, t\right)+\gamma_{1}\left(|| u_{[0, t]}||\right)+\gamma_{2}\left(|| y_{[0, t]}||\right)
$$

is satisfied $\forall x_{0} \in \mathbb{R}^{n}, \forall u \in M_{\mathbb{R}^{m}}$ and all $t \in\left[0, t_{\max }\left(x_{0}, u\right)\right)$. Here, $t_{\max }\left(x_{0}, u\right) \leqslant+\infty$ defines the interval of definition of the solution of system (3.1); as before, $y(t)=h\left[x\left(t, x_{0}, u\right)\right]$ is the output function. 
Definition 3.7 [29]. For system (3.1), the IOSS Lyapunov function is the function $V$ having the following properties:

(1) there exist functions $\alpha_{1}, \alpha_{2} \in \mathcal{K}_{\infty}$ such that

$$
\alpha_{1}(|x|) \leqslant V(x) \leqslant \alpha_{2}(|x|) \quad \forall x \in \mathbb{R}^{n}
$$

is satisfied;

(2) the function $V$ is continuously differentiable along the trajectories of system (3.1), at that there exist functions $\alpha, \sigma_{1}, \sigma_{2} \in \mathcal{K}_{\infty}$ such that

$$
D V(x) f(x, u) \leqslant-\alpha(|x|)+\sigma_{1}(|u|)+\sigma_{2}(|y|) \quad \forall x \in \mathbb{R}^{n}, u \in \mathbb{R}^{m} .
$$

Under the conditions of Definitions 3.6 and 3.7, Theorem 3.2 admits the following extension.

Theorem 3.3 [29]. System (3.1) is IOSS if and only if it has the IOSS Lyapunov function.

Additional equivalent means to define the IOSS property can be found in [34]. Both the OSS and IOSS properties has similar formulations in the form of estimates of the system solutions given by functions from the classes $\mathcal{K} \mathcal{L}$ and $\mathcal{K}$ which follow from the corresponding Lyapunov functions, which allows one to classify these properties with the general ISS concept.

\subsection{Relation between IOSS and Existence of the Observer of the Norm of State Vector}

Apart from the elegant theoretical formulation within the framework of the ISS concept, the IOSS property has an interesting practical aspect associated with existence of the observer of the norm of the state vector for this type of systems.

Definition 3.8 [35]. System $\dot{z}=g(z, y, u), \psi=\kappa(z, y), z \in \mathbb{R}^{l}, g: \mathbb{R}^{l+p+m} \rightarrow \mathbb{R}^{l}, \kappa: \mathbb{R}^{l+p} \rightarrow \mathbb{R}_{+}$ is called the observer of the norm of the state vector for (3.1) if

(a) there exist functions $\beta_{1} \in \mathcal{K} \mathcal{L}$ and $\gamma_{1}, \gamma_{2} \in \mathcal{K}$ such that

$$
\left|\psi\left(t, z_{0}, y\left(t, x_{0}, u\right), u\right)\right| \leqslant \beta_{1}\left(\left|z_{0}\right|, t\right)+\gamma_{1}\left(\|y\|_{[0, t]}\right)+\gamma_{2}\left(\|u\|_{[0, t]}\right)
$$

is satisfied for all $x_{0} \in \mathbb{R}^{n}, z_{0} \in \mathbb{R}^{l}, u \in M_{\mathbb{R}^{m}}$ over the entire interval of definition of solutions;

(b) there exist functions $\beta_{2} \in \mathcal{K} \mathcal{L}$ and $\rho \in \mathcal{K}$ such that

$$
\left|x\left(t, x_{0}, u\right)\right| \leqslant \beta\left(\left|x_{0}\right|+\left|z_{0}\right|, t\right)+\rho\left(\left|\psi\left(t, z_{0}, y\left(t, x_{0}, u\right), u\right)\right|\right)
$$

is satisfied for all $x_{0} \in \mathbb{R}^{n}$ and $z_{0} \in \mathbb{R}^{l}, u \in M_{\mathbb{R}^{m}}$, over the entire interval of definition of solutions.

Theorem 3.4 [35]. System (3.1) admits existence of the observer of the norm of the state vector if and only if it is IOSS.

As was already noted, the IOSS property is a roughened analog of the property of detectability, that is, for the identically zero input and output, the state vector tends to zero, and for bounded input and output the state is bounded as well. According to the last assertion, this property implies that there exists a mechanism for calculation of the numerical estimates of the norm of state (boundedness at the qualitative level follows from the property itself). For example, if the system has an IOSS Lyapunov function from Definition 3.7, then $g(z, y, u)=-\alpha \circ \alpha_{2}^{-1}(z)+\sigma_{1}(|u|)+\sigma_{2}(|y|)$, $\kappa(z, y)=\alpha_{1}^{-1}(z), \rho(s)=s$ can be selected for $z \in \mathbb{R}_{+}$. 


\subsection{Input to Output Stability}

Let us consider a generalization of the ISS property to the systems with output (3.1) (a definition of the property of unboundedness observability (UO) used here is given in Section 6).

Definition 3.9 $[33,36]$. System (3.1) having UO is input to output stable (IOS) if there exist function $\beta \in \mathcal{K} \mathcal{L}$ and $\gamma \in \mathcal{K}$ such that $\forall t \geqslant 0, x_{0} \in \mathbb{R}^{n}, u \in M_{\mathbb{R}^{m}}$

$$
\left|y\left(x_{0}, u, t\right)\right| \leqslant \beta\left(\left|x_{0}\right|, t\right)+\gamma(|| u||)
$$

is satisfied;

if additionally the system is output-Lagrange stable (OL), that is, there exist functions $\sigma_{1}, \sigma_{2} \in \mathcal{K}$ such that

$$
\left|y\left(x_{0}, d, t\right)\right| \leqslant \sigma_{1}\left(\left|h\left(x_{0}\right)\right|\right)+\sigma_{2}(|| u||)
$$

is satisfied for $\forall t \geqslant 0, x_{0} \in \mathbb{R}^{n}$, then the system is called output-Lagrange input to output stable (OLIOS);

if for the system there exist functions $\beta \in \mathcal{K} \mathcal{L}$ and $\gamma \in \mathcal{K}$ such that

$$
\left|y\left(x_{0}, u, t\right)\right| \leqslant \beta\left(\left|h\left(x_{0}\right)\right|, t\right)+\gamma(\|u\|)
$$

is satisfied for $\forall t \geqslant 0, x_{0} \in \mathbb{R}^{n}$, then the system is called state-independent input to output stable (SIIOS);

the system is called robustly output stable (ROS) if for it there are functions $\lambda \in \mathcal{K}_{\infty}$ and $\beta \in \mathcal{K} \mathcal{L}$ such that the system

$$
\dot{x}=g(x, d)=f(x, d \lambda(|y|)), \quad y=h(x)
$$

has the UO property and the estimate

$$
\left|y_{\lambda}\left(x_{0}, d, t\right)\right| \leqslant \beta\left(\left|x_{0}\right|, t\right)
$$

is satisfied $\forall t \geqslant 0, x_{0} \in \mathbb{R}^{n}, d \in M_{B}$, where $B=\left\{d \in \mathbb{R}^{m}:|d| \leqslant 1\right\}$ and $y_{\lambda}\left(x_{0}, d, t\right)$ is the solution in the system output.

For the case of $h(x)=x$, the IOS and SIIOS properties are reduced to ISS. In the case of studying the asymptotic properties of output stability, one must foresee existence of solutions for all $t \geqslant 0$, because in the general case a situation is possible where the output $y(t)$ is bounded or tends to zero, whereas the solution $x(t)$ exists only over a finite interval (it is namely to solve this problem that a weakened analog of the property of extendability of solutions for systems with output, the UO property, was introduced in Definition 3.9). A distinction of IOS from ISS (SIIOS) lies in that for the IOS system it does not follow from smallness of $\left|h\left(x_{0}\right)\right|$ that the general deviation of system $|y(t)|$ is small; it is proportional to the nonestimable value $\left|x_{0}\right|$. For the ISS or SIIOS systems, in the $\mathcal{K} \mathcal{L}$ estimates to the left and right there is the same variable $(x(t)$ or $y(t)$, respectively). In conclusion, the robust output stability of system (3.1) may be commented as follows: the system is uniformly robust stable in output relative to input from $B$ for any output feedback control law bounded by the function $\lambda$ of the system robustness boundary.

Lemma 3.3 [36]. For system (3.1) having the UO property, the following relations are valid:

$$
S I I O S \Rightarrow O L I O S \Rightarrow I O S \Rightarrow R O S \text {. }
$$

In the general case, all inverse relations are not satisfied.

We underline that for the case of stability in state $(h(x)=x)$ SIIOS follows from ROS. 
Definition 3.10 $[37,38]$. For system (3.1), the smooth function $V$ and the function $\lambda: \mathbb{R}^{n} \rightarrow \mathbb{R}_{+}$ are called the IOS Lyapunov function and the auxiliary module if there exist functions $\alpha_{1}, \alpha_{2} \in \mathcal{K}_{\infty}$ satisfying

$$
\alpha_{1}(|h(x)|) \leqslant V(x) \leqslant \alpha_{2}(|x|)
$$

for all $x \in \mathbb{R}^{n}$ and also $\chi \in \mathcal{K}$ and $\alpha_{3} \in \mathcal{K} \mathcal{L}$ such that

$$
V(x)>\chi(|u|) \Rightarrow D V(x) f(x, u) \leqslant-\alpha_{3}(V(x), \lambda(x))
$$

for all $x \in \mathbb{R}^{n}, u \in \mathbb{R}^{m}$. Additionally, for all $u \in M_{\mathbb{R}^{m}}$ and $x_{0} \in \mathbb{R}^{n}$ and some $\delta \in \mathcal{K}$ for any $T \geqslant 0$ :

$$
\begin{gathered}
V\left(x\left(t, x_{0}, u\right)\right)>\chi(|u(t)|), \\
t \in[0, T) \Rightarrow \lambda\left(x\left(t, x_{0}, u\right)\right) \leqslant \max \left\{\delta\left(\left|x_{0}\right|\right), \delta(\| u||)\right\} .
\end{gathered}
$$

The function $V$ is called the OLIOS Lyapunov function if inequality (3.6) can be strengthened to

$$
\alpha_{1}(|h(x)|) \leqslant V(x) \leqslant \alpha_{2}(|h(x)|), \quad \alpha_{1}, \alpha_{2} \in \mathcal{K}_{\infty}
$$

The function $V$ is called the SIIOS Lyapunov function if inequality (3.8) is satisfied for some $\alpha_{1}, \alpha_{2} \in \mathcal{K}_{\infty}$ and there are functions $\chi, \alpha_{3} \in \mathcal{K}$ such that for all $x \in \mathbb{R}^{n}$ and $u \in \mathbb{R}^{m}$ :

$$
V(x)>\chi(|u|) \Rightarrow D V(x) f(x, u) \leqslant-\alpha_{3}(V(x)) .
$$

The function $V$ is called the ROS Lyapunov function if inequality (3.6) is satisfied for some $\alpha_{1}, \alpha_{2} \in \mathcal{K}_{\infty}$ and there are functions $\chi \in \mathcal{K}$ and $\alpha_{3} \in \mathcal{K} \mathcal{L}$ such that for all $x \in \mathbb{R}^{n}$ and $u \in \mathbb{R}^{m}$ :

$$
|y|>\chi(|u|) \Rightarrow D V(x) f(x, u) \leqslant-\alpha_{3}(V(x),|x|) .
$$

The IOS Lyapunov function was introduced in [37] for the bounded input bounded state (BIBS) systems defined in Section 6. In this case, the function $|x|$ can be used as an auxiliary module $\lambda$, and inequality (3.7) is representable as follows:

$$
V(x)>\chi(|u|) \Rightarrow D V(x) f(x, u) \leqslant-\alpha_{3}(V(x),|x|) .
$$

Theorem 3.5 [37, 38]. Let (3.1) be UO system. The following properties are equivalent:

(1) system (3.1) IOS (OLIOS, SIIOS);

(2) system (3.1) has IOS (OLIOS, SIIOS) Lyapunov function and auxiliary module.

We emphasize that in the case of SIIOS there is no need for an auxiliary module.

Theorem 3.6 [37]. Let system (3.1) have the BIBS property. Then, if system (3.1) has IOS (OLIOS, SIIOS, ROS) Lyapunov function with the auxiliary module $|x|$, then system (3.1) is IOS (OLIOS, SIIOS, ROS).

The difference between the IOS and SIIOS properties becomes more evident at comparing the corresponding Lyapunov functions. For SIIOS, the Lyapunov function is bounded from above by the function of distance to the set, whereas for the IOS system the Lyapunov function can grow proportionally to the norm of the state vector. According to the concept of the ISS systems, the property of input to output stability may be characterized in some cases using the asymptotic coefficients. 
Definition 3.11 [39]. System (3.1) having the UO property has asymptotic output gain if for all $u \in M_{\mathbb{R}^{m}}$ and $x_{0} \in \mathbb{R}^{n}$ there exists function $\gamma \in \mathcal{K}$ such that

$$
\varlimsup_{t \rightarrow+\infty}\left|y\left(t, x_{0}, u\right)\right| \leqslant \gamma(\|u\|) .
$$

Theorem 3.7 [39]. System (3.1) having the UO property is IOS if and only if it is OL and has asymptotic output gain.

Stability of the nonlinear systems is a complicated field of study. Therefore, in some case where one fails to construct the desired Lyapunov function it is advisable to use equivalent formulations of stability as asymptotic gains given in Definitions 2.2-3.11.

\subsection{ISS and iISS Properties with Respect to Set}

Extension of the ISS property to the case of compact sets is described in [40-42]. In this case, it is assumed that system (2.3) has a compact set $\mathcal{A}$ that is invariant for the zero input $u$. System (2.3) is called ISS relative to $\mathcal{A}$ if for any input $u \in M_{\mathbb{R}^{m}}$ and $x_{0} \in \mathbb{R}^{n}$ there exist functions $\beta \in \mathcal{K} \mathcal{L}$, $\gamma \in \mathcal{K}$ such that $\forall t \geqslant 0$

$$
\left|x\left(t, x_{0}, u\right)\right|_{\mathcal{A}} \leqslant \beta\left(\left|x_{0}\right|_{\mathcal{A}}, t\right)+\gamma(\|u\|) .
$$

If $\mathcal{A}=\{0\}$, then the last inequality is reduced to the standard estimate from Definition 2.1. For $u=0$, the ISS property with respect to the compact set comes to the property of uniform robust global asymptotic stability (UGAS) introduced in Section 6. The ISS property with respect to the set also is a variant of formulation of the property of practical input to state stability [43] where for all $x_{0} \in \mathbb{R}^{n}, u \in M_{\mathbb{R}^{m}}$ there exist functions $\beta \in \mathcal{K} \mathcal{L}, \gamma \in \mathcal{K}$ and a constant $\varepsilon>0$ such that

$$
\left|x\left(t, x_{0}, u\right)\right| \leqslant \beta\left(\left|x_{0}\right|, t\right)+\gamma(\| u||)+\varepsilon .
$$

It turns out that the ISS Lyapunov function for the compact sets is formulated similar to Definition 2.4 and is an equivalent of ISS relative to the compact $\mathcal{A}$ : the smooth function $V: \mathbb{R}^{n} \rightarrow$ $\mathbb{R}_{+}$is called the ISS Lyapunov function relative to $\mathcal{A}$ if there exist functions $\alpha_{1}, \alpha_{2}, \alpha \in \mathcal{K}_{\infty}$ and $\theta \in \mathcal{K}$ such that the inequalities

$$
\begin{gathered}
\alpha_{1}\left(|x|_{\mathcal{A}}\right) \leqslant V(x) \leqslant \alpha_{2}\left(|x|_{\mathcal{A}}\right), \\
D V(x) f(x, u) \leqslant \theta(|u|)-\alpha\left(|x|_{\mathcal{A}}\right)
\end{gathered}
$$

are satisfied for all $x \in \mathbb{R}^{n}, u \in \mathbb{R}^{m}$.

We notice that the substitution $h(x)=|x|_{\mathcal{A}}$ drives the property ISS for the set $\mathcal{A}$ into the property SIIOS in output $y=h(x)$. We emphasize that SIIOS is not a variant of the ISS property relative to the set in a different notation; it is a more "complex" stability property because in the general case the trend $y(t) \rightarrow 0$ needs not imply for $t \rightarrow+\infty$ that simultaneously $x(t) \rightarrow Z$. The case of noncompact set $\mathcal{A}$ is considered in [44]. For the case of noncompact set $\mathcal{A}$, one has to take into account definiteness of the solutions of system (3.1) for all $t \geqslant 0$ and introduce the requirement for the property of solution extendability or, by analogy with IOS, the UO property. The iISS property for the set was formulated in [45]: system (2.3) is called iISS relative to $\mathcal{A}$ if for any input $u \in M_{\mathbb{R}^{m}}$ and $x_{0} \in \mathbb{R}^{n}$ there are functions $\beta \in \mathcal{K} \mathcal{L}, \alpha, \gamma \in \mathcal{K}_{\infty}$ such that $\forall t \geqslant 0$

$$
\alpha\left(\left|x\left(t, x_{0}, u\right)\right|_{\mathcal{A}}\right) \leqslant \beta\left(\left|x_{0}\right|_{\mathcal{A}}, t\right)+\int_{0}^{t} \gamma(|u(\tau)|) d \tau .
$$

For $\mathcal{A}=\{0\}$, the last inequality is reduced to the estimate from Definition 2.7, and for $u=0$ the property of iISS for the set comes to the property of UGAS. The Lyapunov function for this property

AUTOMATION AND REMOTE CONTROL Vol. 72 No. 82011 
is constructed similar to Definition 3.1: continuously differentiable function $V: \mathbb{R}^{n} \rightarrow \mathbb{R}_{+}$is called the iISS Lyapunov function relative to the set $\mathcal{A}$ if there exist for it the functions $\alpha_{1}, \alpha_{2} \in \mathcal{K}_{\infty}$, $\sigma \in \mathcal{K}$ and positive definite continuous function $\alpha$ such that $\forall x \in \mathbb{R}^{n}, u \in \mathbb{R}^{m}$ :

$$
\begin{gathered}
\alpha_{1}\left(|x|_{\mathcal{A}}\right) \leqslant V(x) \leqslant \alpha_{2}\left(|x|_{\mathcal{A}}\right), \\
D V(x) f(x, u) \leqslant-\alpha\left(|x|_{\mathcal{A}}\right)+\sigma(|u|) .
\end{gathered}
$$

The distinction between the ISS and iISS Lyapunov functions relative to the set lies in the requirements presented to the function $\alpha$ (for the case of ISS, $\alpha \in \mathcal{K}_{\infty}$ ). Existence of the Lyapunov function in the iISS system relative to the compact set $\mathcal{A}$ is a sufficient condition for iISS relative to $\mathcal{A}$. By analogy with Theorem 3.1, one can prove [45] that other sufficient conditions for the iISS property relative to the compact set $\mathcal{A}$ are as follows:

(1) There exists an output $h\left(|h(x)| \leqslant \kappa\left(|x|_{\mathcal{A}}\right), \quad \kappa \in \mathcal{K}_{\infty}\right)$ having the property $x_{0} \in \mathbb{R}^{n}$, $y\left(t, x_{0}, 0\right)=0, t \geqslant 0 \Rightarrow \lim _{t \rightarrow+\infty}\left|x\left(t, x_{0}, 0\right)\right|_{\mathcal{A}}=0$, and there are continuously differentiable function $W: \mathbb{R}^{n} \rightarrow \mathbb{R}_{+}, \alpha_{1}, \alpha_{2} \in \mathcal{K}_{\infty}, \sigma \in \mathcal{K}$ and positive definite function $\alpha_{4}$ such that for all $x \in \mathbb{R}^{n}$, $u \in \mathbb{R}^{m}$

$$
\begin{gathered}
\alpha_{1}\left(|x|_{\mathcal{A}}\right) \leqslant W(x) \leqslant \alpha_{2}\left(|x|_{\mathcal{A}}\right), \\
D W(x) f(x, u) \leqslant-\alpha_{4}(|h(x)|)+\sigma(|u|) .
\end{gathered}
$$

(2) The UGAS property is satisfied for $\mathcal{A}$ for $u=0$, and there are continuously differentiable function $V: \mathbb{R}^{n} \rightarrow \mathbb{R}_{+}, \alpha_{1}, \alpha_{2} \in \mathcal{K}_{\infty}, \sigma \in \mathcal{K}$ such that for all $x \in \mathbb{R}^{n}, u \in \mathbb{R}^{m}$

$$
\begin{gathered}
\alpha_{1}\left(|x|_{\mathcal{A}}\right) \leqslant V(x) \leqslant \alpha_{2}\left(|x|_{\mathcal{A}}\right), \\
D V(x) f(x, u) \leqslant \sigma(|u|) .
\end{gathered}
$$

\subsection{Relation between IOS, ISS, and IOSS Properties}

The IOS property characterizes the "external" stability of system (3.1), that is, stability to the inputs and outputs that are external to the system. The ISS property guarantees "internal" stability of the system or boundedness of the state vector in the presence of bounded input. The property of robust detectability (IOSS), defines intuitively the relation between "external" signals $u, y$ and "internal" state of the system $x$, the relation between the "external" and "internal" stability. This intuitive relation turns out to have a fundamental application.

Theorem 3.8 [43]. System (3.1) is ISS if and only if it is IOSS and IOS.

We complete cataloguing the properties established within the framework of the ISS concept and proceed to representing the results of practical application of the theory. A more detailed list of various properties accompanying ISS is given in the review [2].

\section{CONNECTIONS OF THE ISS, IISS, AND LISS SYSTEMS}

The ISS concept proves convenient for studying various connections of the nonlinear systems, which, in particular, enables one to study systems of high dimensionalities. Let us consider $n$ equations

$$
\dot{x}_{i}=f_{i}\left(x_{1}, \ldots, x_{n}, u_{i}\right), \quad i=1, \ldots, n,
$$

where for the $i$ th equation $x_{i} \in \mathbb{R}^{N_{i}}$ is the state and $x_{j} \in \mathbb{R}^{N_{j}}, j \neq i$, and $u_{i} \in \mathbb{R}^{M_{i}}$ are the input. By denoting $N=\sum_{i=1}^{n} N_{i}, M=\sum_{i=1}^{n} M_{i}, x=\left(x_{1}^{\mathrm{T}}, \ldots, x_{n}^{\mathrm{T}}\right)^{\mathrm{T}}, u=\left(u_{1}^{\mathrm{T}}, \ldots, u_{n}^{\mathrm{T}}\right)$ and introducing in 
a corresponding manner $f: \mathbb{R}^{N+M} \rightarrow \mathbb{R}^{N}$, one can set down the assembly of Eqs. (4.1) as a single system uniting $n$ subsystems:

$$
\dot{x}=f(x, u) \text {. }
$$

By the use of the inverse reasoning one often can represent a high-dimensionality system (4.2) as a connection of several systems of lower dimensionalities (4.1). This gives rise to the question as to whether it is possible to draw a conclusion about stability of the entire system (4.2) from the ISS/iISS properties of each of its subsystems in (4.1). Analysis or synthesis of the ISS/iISS property for the reduced systems (4.1) of smaller dimensionality may sometimes have a simpler solution than for (4.2). A network or graph whose vertices are individual systems $i=1, \ldots, n$ and an edge directed from vertex $i$ to vertex $j$ belongs to the graph if and only if $x_{i}$ is the input of system $j$, that is, $f_{j}$ depends explicitly on $x_{i}$, or lacks, otherwise, can be assigned to each such connected system. We assume that each system (4.1) is ISS, that is,

$$
\left|x_{i}(t)\right| \leqslant \max \left\{\beta\left(\left|x_{i}^{0}\right|, t\right), \max _{j=1, \ldots, n} \gamma_{i j}\left(\| x_{j}||\right), \gamma_{i}(\|u\|)\right\}
$$

is satisfied for any input $x_{j}, i \neq j=1, \ldots, n, u_{i} \in \mathbb{R}^{M_{i}}$ and initial state $x_{i}^{0}$. We recall that if in this inequality the maxima of the corresponding functions are replaced by their sum, then an equivalent definition of ISS results. What can be said about stability of the entire system (4.2)? It turns out that serial connection of ISS systems is always ISS. This can be readily demonstrated for two systems by using the definition of ISS for trajectories and substituting one estimate into another. Additionally, generation of the Lyapunov function for such connection presents no difficulties if the Lyapunov functions are given for each of the subsystems. This reasoning is easily extended to the case of an arbitrary structure of serial connection of $n \geqslant 2$ systems, that is, a tree-like connection whose graph has no cycles. In the case of feedback connection, stability may be violated. The conditions for gain smallness

$$
\gamma_{12} \circ \gamma_{21}(s)<s \quad \forall s>0,
$$

meaning that the composition of these functions is a compressing map were developed to establish stability of two feedback systems [43]. If in the definition of ISS the impact of input is reflected in the sum of gains, rather than in the maximum of these functions, then the small gain condition is given by [46]:

$$
\left(s+\alpha_{1}(s)\right) \circ \gamma_{12} \circ\left(s+\alpha_{2}(s)\right) \circ \gamma_{21}(s)<s \quad \forall s>0,
$$

where arbitrary $\alpha_{1}, \alpha_{2}$ can be taken from the class of $\mathcal{K}$-functions. Additionally, if this condition is satisfied, then the Lyapunov function can be generated explicitly as a special combination of the Lyapunov functions of the subsystems [46]. These results were extended to the case of two iISS systems [47], as well as for the connection of discrete systems, delay systems, hybrid systems, and a class of systems that do not satisfy the semigroup property [14]. In the case of arbitrary connection of ISS systems $(n \geqslant 2)$, the total number of the gains of the entire connection grows in proportion to $n^{2}$. These gains can be conveniently compiled as a matrix

$$
\Gamma:=\left[\begin{array}{cccc}
0 & \gamma_{12} & \cdots & \gamma_{1 n} \\
\gamma_{21} & 0 & \ddots & \gamma_{2 n} \\
\vdots & \ldots & \ddots & \vdots \\
\gamma_{n 1} & \gamma_{n 2} & \cdots & 0
\end{array}\right],
$$


and in the case of a definition with maximum in gains the nonlinear operator can be defined depending on the definition of ISS used as

$$
\Gamma_{\max }\left(s_{1}, \ldots, s_{n}\right)^{\mathrm{T}}:=\left(\max _{j=1, \ldots, n} \gamma_{1 j}\left(s_{j}\right), \ldots, \max _{j=1, \ldots, n} \gamma_{n j}\left(s_{j}\right)\right)^{\mathrm{T}}
$$

or as

$$
\Gamma_{\sum}\left(s_{1}, \ldots, s_{n}\right)^{\mathrm{T}}:=\left(\sum_{j=1}^{n} \gamma_{1 j}\left(s_{j}\right), \ldots, \sum_{j=1}^{n} \gamma_{n j}\left(s_{j}\right)\right)^{\mathrm{T}}
$$

if the definition uses the sum of gains. We introduce the following notation to formulate further results:

$$
a>b \Leftrightarrow a_{i}>b_{i} \quad \forall i=1, \ldots, n .
$$

The relations $a<b, a \leqslant b, a \geqslant b$ are defined similarly. The logic negation of the last relation is denoted by $a \nsupseteq b$ which means that there is a subscript $i$ such that $a_{i}<b_{i}$.

Theorem 4.1 [48]. Let each of the systems (4.1) be ISS with gains from (4.3). If

$$
\Gamma_{\max }(s) \ngtr s \quad \forall s \in \mathbb{R}_{+}^{n} \backslash\{0\}
$$

is satisfied, then the connection of these systems (4.2) is ISS.

Condition (4.9) is called the gain smallness condition. In the case of using the definition of ISS with summation of gains, this condition is replaced by

$$
D \circ \Gamma_{\sum}(s) \ngtr s \quad \forall s \in \mathbb{R}_{+}^{n} \backslash\{0\},
$$

where $D(s):=\left(s_{1}+\alpha\left(s_{1}\right), \ldots, s_{n}+\alpha\left(s_{n}\right)\right)^{\mathrm{T}}$ and $\alpha$ is an arbitrary $\mathcal{K}$-function. If the ISS of the Lyapunov function are known for each connection system and the corresponding gains satisfy the smallness condition, then it is possible to construct auxiliary scaling functions $\sigma_{i} \in \mathcal{K}, i=1, \ldots, n$, such that for any $t>0[9,49]$

$$
\Gamma_{\max }(\sigma(t))<\sigma(t), \quad \sigma(t):=\left(\sigma_{1}(t), \ldots, \sigma_{n}(t)\right)^{\mathrm{T}}
$$

and the ISS Lyapunov function of the entire connection can be taken as

$$
V(x)=\max _{i}\left\{\sigma_{1}^{-1}\left(V_{1}\left(x_{1}\right)\right), \ldots, \sigma_{n}^{-1}\left(V_{n}\left(x_{n}\right)\right)\right\} .
$$

Various applications of these results to the studies of large systems for stability can be found in $[9,50]$. In some applications, the ISS property and even a weaker iISS property can turn out to be rather restrictive. Therefore, sometimes it makes sense to consider a wider class of LISS systems. Large connections of such systems were discussed in [51]. In this case, to provide connection stability, the gain smallness condition is replaced by its local version, that is,

$$
\Gamma(s) \nsupseteq s \quad \forall 0 \leqslant s \leqslant w^{*}, \quad s \neq 0 \quad \text { and } \quad \Gamma\left(w^{*}\right)<w^{*}
$$

must be satisfied for some $w^{*} \in \mathbb{R}_{+}^{n}$.

It is noteworthy that the local version of the stability condition is independent of whether gain summation or their maximum is used in the definition of LISS. Therefore, in (4.13) the subscript $\sum$ or max of the operator $\Gamma$ is omitted. The Lyapunov function can be formed similar to the case of connection of the ISS systems. Estimates of the constraints on the local conditions and inputs for the LISS property of the entire connection which depend not only on the initial constraints for the subsystems, but also on the vector $w^{*}$ included in condition (4.13) were given in [51]. 


\section{USE OF THE ISS CONCEPT FOR DESIGN AND ANALYSIS OF THE CONTROL SYSTEMS}

We touch upon some aspects of using the described family of properties of robust stability for different types of the nonlinear dynamic systems.

\subsection{ISS Property for the Lur'e Systems}

The Lur'e system is a linear system closed by the nonlinear output feedback

$$
\dot{x}=A x+B(d-\phi(y)), \quad y=C x,
$$

where all matrices of the corresponding dimension have real constant coefficients, $x \in \mathbb{R}^{n}, y \in \mathbb{R}^{m}$, and $d \in \mathbb{R}^{m}$ are, respectively, the vectors of state, output, and perturbation.

Theorem 5.1 [52]. Let the pair of matrices $(C, A)$ of system (5.1) be observable and there exist a matrix $P=P^{\mathrm{T}} \geqslant 0$ such that $A^{\mathrm{T}} P+P A \leqslant 0, P B=C^{\mathrm{T}}$. If there exist $\mu>0$ and $\lambda \in \mathcal{K}_{\infty}$ such that $|y| \lambda(|y|) \leqslant y^{\mathrm{T}} \phi(y)$ for all $y \in \mathbb{R}^{m},|\phi(y)| \leqslant y^{\mathrm{T}} \phi(y)$ for all $|y| \geqslant \mu$, then the system is ISS in the input $d$.

We note that similar results were obtained earlier (see, for example, [53]) in terms of boundedness of solutions under bounded input and asymptotic stability under zero perturbation, which, according to Theorem 2.1 is equivalent to the property of ISS. As in the classical results of the theory of absolute stability [54,55], certain sector constraints on growth of the function $\phi$ are imposed to prove ISS. We accentuate that the conditions of Theorem 5.1 concerned with existence of the matrix $P$ are not conservative (for example, the additional conditions $P>0, A^{\mathrm{T}} P+P A<0$ imply passivity of the linear part of system $(5.1)[56,57]$ (see Definition 6.4), in this case the choice of $\phi(y)=k \operatorname{sgn}(y)$ provides global stabilization of the system uniformly in all $|d| \leqslant k)$.

\subsection{ISS and iISS of the Passive Systems}

The properties of passivity and strict passivity of systems (2.4) and (2.3) are introduced in Definition 6.4 in Section 6. Their popularity accounts for their importance. Any system (2.4) with the Lyapunov function $V$ which is asymptotically stable for $u=0$ is strictly passive in output

$h(x)=\left[\frac{\partial V}{\partial x} G(x)\right]^{\mathrm{T}}=L_{G} V(x)$. A large class of mechanical and physical systems consists of passive systems using the function of system total energy as the supply rate $V[17,57]$. We recall that the property of asymptotic stability under zero input needs not be retained even under the action of a vanishing perturbation. This fact accounts for the importance of analysis of the robust properties for the (strictly) passive systems and the need for the ISS/iISS control laws in the applications. The following lemma lists conditions for the strictly passive systems which make them robust in $L_{2}$ inputs without additional control.

Lemma 5.1 [58]. Let system (2.4) be strictly passive with differentiable positive definite and proper unbounded supply rate $V$, and let any of the following conditions be satisfied:

(a) $\lim _{|x| \rightarrow+\infty} \frac{|h(x)|}{V(x)}<+\infty$;

(b) $\left|\frac{\partial V}{\partial x} G(x)\right| \leqslant b[V(x)]$ for all $x \in \mathbb{R}^{n}$, where $b: \mathbb{R}_{+} \rightarrow \mathbb{R}_{+}$is a positive definite function and

$$
\int_{0}^{+\infty} \frac{d r}{1+b(r)}=+\infty .
$$

Then, the strictly passive system (2.4) is iISS in input u. 
We return to the problem robust stabilization. The control law

$$
u=-\varphi(y)+v
$$

where $\varphi: \mathbb{R}^{m} \rightarrow \mathbb{R}^{m}$ is a continuous function, $y^{T} \varphi(y)>0$ for all $y \neq 0, v \in \mathbb{R}^{m}$ is a new perturbation vector robustness to which must be verified, is known [24] to endow the passive system (2.4) with global asymptotic stability for $v=0$, provided that the system is detectable at zero in the output $y$ (Definition 3.2). Therefore, in what follows we analyze the advantages of the control (5.2).

Lemma 5.2 [58]. Let system (2.4) be strictly passive with differentiable positive definite and proper unbounded supply rate $V$. Then, control (5.2) provides iISS in $v$ if $y^{\mathrm{T}} \varphi(y) \geqslant \varepsilon|y|^{2}, \varepsilon>0$, for all $y \in \mathbb{R}^{m}$. If in Definition 6.4 the function $\beta \in \mathcal{K}_{\infty}$, then control (5.2) provides system (2.4) with the ISS property, at that $V$ is the corresponding ISS Lyapunov function.

Lemma 5.2 establishes the relation between the form of the function $\beta$ and robustness of system (2.4), (5.2) in inputs from $L_{\infty}$ or $L_{2}$. The following is an important consequence of the lemma: a strictly passive systems can have the ISS/iISS property with an arbitrarily small output feedback gain ( $\varepsilon$ may be selected arbitrarily). We notice that control (5.2) was designed using the method of speed gradient [57]. Now we consider the problem of ISS/iISS stabilization for the passive systems,

Theorem 5.2 [58]. Let system (2.4) be passive with differentiable positive definite and proper unbounded supply rate $V$. Control (5.2) provides this system with

(a) the iISS property if the system is detectable at zero in the output $h(x)=L_{G} V(x)$ and either condition (a) or (b) of Lemma 3.1 is satisfied;

(b) the ISS property if the inequality $\theta(|x|) \leqslant|h(x)|$ is true for all $x \in \mathbb{R}^{n}$ and some $\theta \in \mathcal{K}_{\infty}$, and $y^{\mathrm{T}} \varphi(y) \geqslant \varepsilon|y|^{2}, \varepsilon>0$, for all $y \in \mathbb{R}^{m}$.

Item (a) of Theorem 5.2 extends the result of [24] on the asymptotic stabilization of the passive systems to the iISS stabilization.

\subsection{ISS/IOS Stabilization with the Use of Backstepping}

Backstepping (the method of reverse detour of the integrator) is one of the most popular methods of design of the nonlinear control laws [59]. The problem of ISS and iISS stabilization with the use of backstepping was solved in [25]. An independent version of backstepping was obtained in [60] under the name of the method of analytical design of the aggregated controllers. This method forms the basic analytical approach to the control algorithms in the synergetic control theory proposed in [60] (see also the monograph [57] and review [61]).

In this case consideration is given to the modified system (2.4):

$$
\begin{gathered}
\dot{x}=f(x)+G_{1}(x) z+G_{2}(x) d, \\
\dot{z}=u+F(x, z) d,
\end{gathered}
$$

where $x \in \mathbb{R}^{n}$ and $z \in \mathbb{R}^{m}$ are, respectively, the state vectors of systems (5.3) and (5.4), $u \in \mathbb{R}^{m}$ and $d \in \mathbb{R}^{d}$ are the vectors of control and perturbation, all functions in the right-hand sides of (5.3), (5.4) are assumed to be continuous and locally Lipschitzian, $f(0)=0$. It is assumed that there exists a smooth control law $k: \mathbb{R}^{n} \rightarrow \mathbb{R}^{m}$ such that with substitution of $z=k(x)$ in Eq. (5.3) the system becomes ISS (iISS) in state $x$ and input $d$. It is required to "transfer" this control law through the integrator and suggest a new smooth control $u=\widehat{k}(x, z)$ rendering the property of ISS (iISS) to the entire system (5.3), (5.4). Since system (5.3) with the control law $z=k(x)$ is ISS (iISS), there necessarily exists a corresponding smooth Lyapunov function $W: \mathbb{R}^{n} \rightarrow \mathbb{R}_{+}$such that 
under all $x \in \mathbb{R}^{n}$ and $d \in \mathbb{R}^{d}$

$$
\begin{gathered}
\alpha_{1}(|x|) \leqslant W(x) \leqslant \alpha_{2}(|x|), \\
D W(x)\left[f(x)+G_{1}(x) k(x)+G_{2}(x) d\right] \leqslant-\alpha(|x|)+\sigma(|d|)
\end{gathered}
$$

for some functions $\alpha_{1}, \alpha_{2}, \alpha \in \mathcal{K}_{\infty}, \sigma \in \mathcal{K}$ (for the case of iISS the function $\alpha$ is positive definite). We follow $[25,59]$ and select for system (5.3), (5.4) an ISS (iISS) Lyapunov function as $V(x, z)=$ $W(x)+\frac{1}{2}|z-k(x)|^{2}$, its total time derivative being given by

$$
\begin{gathered}
\dot{V}=D W(x)\left[f(x)+G_{1}(x) z+G_{2}(x) d\right] \\
+[z-k(x)]\left[u+F(x, z) d-D k(x)\left\{f(x)+G_{1}(x) z+G_{2}(x) d\right\}\right] \\
\leqslant-\alpha(|x|)+\sigma(|d|)+[z-k(x)]\left[u+D W(x) G_{1}(x)+F(x, z) d\right. \\
\left.-D k(x)\left\{f(x)+G_{1}(x) z+G_{2}(x) d\right\}\right] .
\end{gathered}
$$

Then, by direct calculations we can demonstrate that $\dot{V} \leqslant-\alpha(|x|)-[z-k(x)]^{2}+\sigma(|d|)+|d|^{2}$ is satisfied for the control $u=D k(x)\left\{f(x)+G_{1}(x) z\right\}-D W(x) G_{1}(x)-[z-k(x)]\left[1+|F(x, z)|^{2}+\right.$ $\left|D k(x) G_{2}(x)\right|^{2}$, which renders to (5.3), (5.4) the property of ISS (iISS). For the case of IOS stabilization, we consider the following variant of system (5.3), (5.4):

$$
\begin{gathered}
\dot{x}=f\left(x, z, v_{1}\right), \quad y=h(x), \\
\dot{z}=u+v_{2},
\end{gathered}
$$

where $x \in \mathbb{R}^{n}$ is the state vector of system (5.5). It is required to stabilize the system with respect to the set $Z=\{x: h(x)=0\}$ defined by the zeros of the output function $y \in \mathbb{R}^{p} ; z \in \mathbb{R}^{m}$ is the state vector of system (5.6); $u \in \mathbb{R}^{m}$ is the control vector; $v_{1} \in \mathbb{R}^{q_{1}}$ and $v_{2} \in \mathbb{R}^{q_{2}}$ are the vectors of external perturbations, $v=\left(v_{1}, v_{2}\right) \in \mathbb{R}^{q}, q=q_{1}+q_{2}$. The functions $h: \mathbb{R}^{n} \rightarrow \mathbb{R}^{p}$ and $f: \mathbb{R}^{n+m+q_{1}} \rightarrow \mathbb{R}^{n}$ are continuous and locally Lipschitzian, $f(0,0,0)=0$. We assume accessibility of the continuously differentiable control law $k: \mathbb{R}^{n} \rightarrow \mathbb{R}^{m}$ such that the system

$$
\dot{x}=f\left(x, k(x)+e, v_{1}\right)
$$

has property IOS in output $y$ and input $v_{1}$ for $e=0$, where the variable $e=z-k(x)$ defines the error of realization of the "virtual" control $k$ (in terms of [60], $e$ is the aggregated macrovariable). It is desired to design with allowance for the control $k$ the control law $u=U(x, z)$ providing IOS from the input $v$ to the output $y$ for the entire system (5.5), (5.6).

Theorem 5.3 [45]. Let system (5.7) have the UO property and there exist continuously differentiable functions $V: \mathbb{R}^{n} \rightarrow \mathbb{R}_{+}$and $k: \mathbb{R}^{n} \rightarrow \mathbb{R}^{m}$ such that for all $x \in \mathbb{R}^{n}, e \in \mathbb{R}^{m}$ and $v_{1} \in \mathbb{R}^{q_{1}}$ :

$$
\begin{aligned}
\alpha_{1}(|h(x)|) & \leqslant V(x) \leqslant \alpha_{2}(|x|), \quad \alpha_{1}, \alpha_{2} \in \mathcal{K}_{\infty} ; \\
\frac{\partial V}{\partial x} f\left(x, k(x), v_{1}\right) & \leqslant-\alpha_{3}(V(x))+\sigma_{1}\left(\left|v_{1}\right|\right)
\end{aligned}
$$

$\alpha_{3} \in \mathcal{K}_{\infty}, \sigma_{1} \in \mathcal{K}$. There are continuous functions $r: \mathbb{R}^{n+m} \rightarrow \mathbb{R}^{m}, b: \mathbb{R}^{n+m} \rightarrow \mathbb{R}_{+}, \lambda \in \mathcal{K}$ such that for all $x \in \mathbb{R}^{n}, z, z^{\prime} \in \mathbb{R}^{m}$ and $v_{1}, v_{1}^{\prime} \in \mathbb{R}^{q_{1}}$

$$
\begin{aligned}
& \frac{\partial V}{\partial x} f\left(x, z, v_{1}\right)-\frac{\partial V}{\partial x} f\left(x, z^{\prime}, v_{1}\right) \leqslant r\left(x, z^{\prime}\right)^{\mathrm{T}}\left(z-z^{\prime}\right) \\
& \frac{\partial k}{\partial x} f\left(x, z, v_{1}\right)-\frac{\partial k}{\partial x} f\left(x, z, v_{1}^{\prime}\right) \leqslant b(x, z) \lambda\left(\left|v_{1}-v_{1}^{\prime}\right|\right) .
\end{aligned}
$$


Then, system (5.5), (5.6) with the control

$$
u=\frac{\partial k}{\partial x} f(x, z, 0)-r(x, k(x))-\varphi(z-k(x))-\frac{1}{2}\left(1+b(x, z)^{2}\right)(z-k(x))
$$

is IOS if $\varphi: \mathbb{R}^{m} \rightarrow \mathbb{R}^{m}$ is a continuous function and $z^{\mathrm{T}} \varphi(z) \geqslant \kappa(|z|)$ for all $z \in \mathbb{R}^{m}, \kappa \in \mathcal{K}_{\infty}$.

(2) (5.8) and (5.9) are satisfied and

$$
\frac{\partial V}{\partial x} f\left(x, k(x)+e, v_{1}\right) \leqslant-\alpha_{4}(V(x))+\sigma_{2}(|e|)+\sigma_{2}\left(\left|v_{1}\right|\right),
$$

$\alpha_{4} \in \mathcal{K}_{\infty}, \sigma_{2} \in \mathcal{K}$. Then, system (5.5), (5.6) with the control

$$
u=\frac{\partial k}{\partial x} f(x, z, 0)-\varphi(z-k(x))-\frac{1}{2}\left(1+b(x, z)^{2}\right)(z-k(x))
$$

has the IOS property, where $\varphi: \mathbb{R}^{m} \rightarrow \mathbb{R}^{m}$ is a continuous function and $z^{\mathrm{T}} \varphi(z) \geqslant \kappa(|z|)$ for all $z \in \mathbb{R}^{m}, \kappa \in \mathcal{K}_{\infty}, \kappa(s) \geqslant \tilde{\kappa}(s)+\sigma_{2}(s), \tilde{\kappa} \in \mathcal{K}_{\infty}$.

The first part of Theorem 5.3 extends the result on ISS/iISS stabilization by backstepping of system (5.3), (5.4) to the case of input to output stability, whereas the second part of the theorem extends the ideas of the method of analytical design of aggregated controllers [60] to the problem of IOS stabilization. Extension of the result of Theorem 5.3 to the case of adaptive input to output stabilization is presented in [62].

\subsection{Universal Laws of ISS, iISS, and IOS Stabilization}

It is common knowledge that under certain constraints the existence of system's Lyapunov function with the given properties amounts to its asymptotic stability. Unfortunately, practical application of this theory is hindered by the lack of general methods for construction of the Lyapunov function for the selected nonlinear system. The problem is simplified with appearance of control in the right-hand side of the dynamic system. In this case, the desired Lyapunov function may be assigned by the choice of the control law. A question arises: which method to use for calculation of control and which Lyapunov function is permissible (assignable) for the system? The backstepping considered in the last section has strict restrictive conditions for applicability. Does this imply that it cannot be used for the selected system and/or the Lyapunov function or that any other method cannot establish the solution? This question is answered by the theory of control Lyapunov functions (CLF) [63-66] (see also the monograph [56]). The necessary and sufficient conditions for the given Lyapunov function to be assigned for the system with the use of some almost smooth (continuous everywhere and smooth outside the origin) control law were proposed in [64]. A variant of the control law solving this problem was suggested in [64].

Definition 5.1 [64]. The differentiable positive definite and proper unbounded function $V$ : $\mathbb{R}^{n} \rightarrow \mathbb{R}_{+}$is called the CLF for system (2.4) if for any $|x| \neq 0$ there exists $u \in \mathbb{R}^{m}$ such that

$$
\frac{\partial V(x)}{\partial x}[f(x)+G(x) u]<0
$$

The function $V$ has the small control property for system (2.4) if

$$
\limsup _{|x| \rightarrow 0} \frac{L_{f} V(x)}{\left|L_{G} V(x)\right|} \leqslant 0 .
$$


Theorem 5.4 [64]. The differentiable positive definite and proper unbounded function $V: \mathbb{R}^{n} \rightarrow$ $\mathbb{R}_{+}$is CLF for system (2.4) if

$$
L_{G} V(x) \equiv 0 \Rightarrow L_{f} V(x)<0
$$

is satisfied for all $|x| \neq 0$. Additionally, for the CLF of the function $V$ the control law

$$
\begin{gathered}
u=-\kappa\left(L_{f} V(x),\left|L_{G} V(x)\right|\right) L_{G} V(x)^{\mathrm{T}}, \\
\kappa(s, r)= \begin{cases}\left(s+\sqrt{s^{2}+b^{4}}\right) r^{-2}, & \text { if } r \neq 0 \\
0, & \text { if } r=0,\end{cases}
\end{gathered}
$$

stabilizes system (2.4) globally and asymptotically. Control (5.11) is a continuous function (almost smooth if all $f, G$ and $V$ are smooth) if the function $V$ features smallness of controls.

We emphasize that if there exists a control law $u=u(x)$ assigning to the system a Lyapunov function $V$, then (5.10) is necessarily satisfied, and, consequently, control (5.11) also stabilizes the system. In virtue of this property, the control law (5.11) is referred to as "universal." It also deserves mentioning that algorithm (5.11) is a special case of the $L_{G} V$ control laws (method of speed gradient). Extension of the CLF theory to the problem of ISS, iISS, and IOS stabilization is presented in [67-69] for which purpose consideration is given to a perturbed modification of the following system (2.4):

$$
\dot{x}=f(x, v)+G(x) u, \quad y=h(x),
$$

where $x \in \mathbb{R}^{n}, u \in \mathbb{R}^{m}, y \in \mathbb{R}^{p}$, and $v \in \mathbb{R}^{r}$ are, respectively, the vectors of state, input, output, and external perturbation; $f, h$, and the columns of the matrix function $G$ are continuous and locally Lipschitzian vector functions, $h(0)=0, f(0,0)=0$.

Definition 5.2. For system (5.13), we call the positive definite and proper unbounded differentiable function $V: \mathbb{R}^{n} \rightarrow \mathbb{R}_{+}$ISS (iISS) CLF if there exist a function $\chi \in \mathcal{K}_{\infty}$ and $\alpha \in \mathcal{K}_{\infty}$ (positive definite function $\alpha: \mathbb{R}_{+} \rightarrow \mathbb{R}_{+}$) such that for all $x \in \mathbb{R}^{n}$ and $v \in \mathbb{R}^{r}$

$$
\inf _{u \in \mathbb{R}^{m}}\{a(x, v)+B(x) u\}<-\alpha(|x|)+\chi(|v|),
$$

where $a(x, v)=D V(x) f(x, v), B(x)=D V(x) G(x)$.

Theorem 5.5. If ISS (iISS) CLF $V$ for system (5.13) has small controls, then the control

$$
u=\kappa\left(\omega(x),|B(x)|^{2}\right) B(x)^{\mathrm{T}}, \quad \mu(x)+\alpha(|x|) / 3 \leqslant \omega(x) \leqslant \mu(x)+2 \alpha(|x|) / 3,
$$

$\mu(x)=\sup _{v \in \mathbb{R}^{r}}\{a(x, v)-\chi(|v|)\}$, where the function $\kappa(s, r)$ is defined in (5.11), is continuous on $\mathbb{R}^{n}$ and makes system (5.13), (5.14) ISS (iISS) for $v \in M_{\mathbb{R}^{r}}$.

For the case of $v=0$, the result of Theorem 5.5 is reduced to the statement of Theorem 5.4.

Definition 5.3. For the system (5.13), we call the differentiated function $V: \mathbb{R}^{n} \rightarrow \mathbb{R}_{+}$IOS CLF is called for the controls $u \in \mathbb{R}^{m}$ if the following conditions are satisfied:

(1) There exist $\alpha_{1}, \alpha_{2} \in \mathcal{K}_{\infty}$ such that (3.6) is true for all $x \in \mathbb{R}^{n}$;

(2) There exists a function $\chi \in \mathcal{K}_{\infty}$ such that

$$
V(x)>\chi(|v|) \Rightarrow a(x, v) \leqslant \psi(x), \quad \psi \in C_{0},
$$

and for all $x \notin Z$

$$
\inf _{u \in \mathbb{R}^{m}}\{\psi(x)+B(x) u\}<0,
$$

AUTOMATION AND REMOTE CONTROL Vol. 72 No. 82011 
where $a(x, v)=D V(x) f(x, v), B(x)=D V(x) G(x)$. If, instead of (3.6), true is (3.8), then such function is called the OLIOS CLF for the controls $u \in \mathbb{R}^{m}$. The function $V$ is said to have the property of control smallness in the output $y$ if for any $\varepsilon>0$ there exists $\delta>0$ such that for all $h(x) \neq 0,|h(x)|<\delta$ there exists a control $u \in \mathbb{R}^{m}$ with $|u|<\varepsilon$ and $\psi(x)+B(x) u<0$.

The second condition of the last definition can be rearranged in a more constructive way as

$$
\begin{gathered}
V(x)>\chi(|v|) \Rightarrow a(x, v) \leqslant \psi(x), \quad \psi \in C_{0} \\
|B(x)|=0 \Rightarrow \psi(x)<0 \quad \forall x \notin Z .
\end{gathered}
$$

Expressions (5.15) and (5.16) define the basic requirements on IOS or OLIOS CLF systems (5.13). The property of global stability modulo output (GSMO) that is used in what follows will be introduced in Section 6.

Theorem 5.6. If IOS (OLIOS) CLF V for system (5.13) has the property of smallness in output $y$, then the control

$$
u=\kappa\left(\psi(x),|B(x)|^{2}\right) B(x)^{\mathrm{T}},
$$

where the function $\kappa(s, r)$ is defined in (5.11), is continuous on $\mathbb{R}^{n}$, and provides to the GSMO system (5.13), (5.17) the property of IOS (OLIOS) for $v \in M_{\mathbb{R}^{r}}$.

For the case of $h(x)=x$, the GSMO property may be omitted, and the conditions of Theorem 5.6 coincide with the corresponding conditions of Theorem 5.5.

\subsection{Design of Robust Observers for Nonlinear Systems}

Let us consider application of the ISS property not only in the problem of stabilization, but also in the problem of observation of the state vector of nonlinear systems. Let [70]

$$
\dot{x}=A x+f(x)+D_{1} w, \quad y=C x+D_{2} w,
$$

where $x \in \mathbb{R}^{n}$ is the state vector, $y \in \mathbb{R}^{p}$ is the measurement vector, $w \in \mathbb{R}^{q}$ is the vector of external perturbations and measurement noise, $f: \mathbb{R}^{n} \rightarrow \mathbb{R}^{n}$ is a locally Lipschitzian continuous function, all matrices are real and have corresponding dimensions, the pair $(A, C)$ is observable. It is assumed that the solutions of this system are defined for all $t \geqslant 0$. The standard observer of the state vector $x$ for system (5.18) is as follows:

$$
\dot{\hat{x}}=A \hat{x}+f(\hat{x})+L(y-C \hat{x}),
$$

where $\hat{x} \in \mathbb{R}^{n}$ is the estimate vector for $x$ and $L$ is the observer matrix. It is required to determine the conditions for the matrix $L$ guaranteeing the SIIOS property to system (5.18), (5.19) in output $e=x-\hat{x}$ (measurement error) and input $w$.

Proposition 5.1 [70]. Let $x(t) \in X \subset \mathbb{R}^{n}$ for all $t \geqslant 0$ and $|f(x)-f(\hat{x})| \leqslant k_{f}|x-\hat{x}|$ for all $x \in X, \hat{x} \in X$ and some $k_{f}>0$. If there exists $P=P^{\mathrm{T}}>0$ and the matrix $L$ such that

$$
\left[\begin{array}{cc}
(A-L C)^{\mathrm{T}} P+P(A-L C)+2 \alpha k_{f} I & P \\
P & -\frac{1}{2} \alpha I
\end{array}\right]<0
$$

for some $\alpha>0$ ( $I$ is the identity matrix of corresponding dimension), then system (5.18), (5.19) has the property of SIIOS in output e and input $w$.

It is proposed in Proposition 5.1 to seek the matrix $L$ as a solution to the linear matrix inequality providing boundedness of the measurement error for any bounded perturbation and measurement noise. Asymptotic observation of the state $x$ by the observer (5.19) is guaranteed in the absence of $w$. 


\section{AUXILIARY INFORMATION}

Let $\mathbb{R}$ be the set of real numbers and $\mathbb{R}_{+}=\{\tau \in \mathbb{R}: \tau \geqslant 0\}$, the set of nonnegative real numbers. The distance in $\mathbb{R}^{n}$ from the given point $x$ to the set $A$ is denoted by $|x|_{A}=\operatorname{dist}(x, A)=$ $\inf _{\eta \in A}|x-\eta|$. Then, $|x|_{\{0\}}=|x|$ is an ordinary Euclidean norm. We denote by $\|u\|_{\left[t_{0}, t\right]}$ the $L_{\infty}$-norm of the Lebesgue-measurable locally bounded almost everywhere function $u: \mathbb{R}_{+} \rightarrow \mathbb{R}^{m}$ :

$$
\|u\|_{\left[t_{0}, t\right]}=\operatorname{ess} \sup \left\{|u(t)|, t \in\left[t_{0}, T\right]\right\} .
$$

If $T=+\infty$, then we just write $\|u\|$. We denote by $M_{\mathbb{R}^{m}}$ the set of all such Lebesgue-measurable functions $u$ having the property $\|u\|<+\infty$ and by $M_{\Omega}$ the set of functions satisfying $u(t) \in \Omega \subset \mathbb{R}^{m}$ almost for all $t \geqslant 0$, where $\Omega$ is a compact set. The function $V: \mathbb{R}^{n} \rightarrow \mathbb{R}_{+}$is called positive definite function if it vanishes only at the origin, and proper unbounded if $V(x) \rightarrow+\infty$ for $|x| \rightarrow+\infty$. By $D V(x) F(\cdot)$ we denote the derivative of the function $V$, if differentiable, in the line of the vector field $F$ and the Dini derivative for continuous and locally Lipschitzian function $V$ :

$$
D V(x) F(\cdot)=\lim _{t \rightarrow 0^{+}} \inf \frac{V[x+t F(\cdot)-V(x)]}{t} .
$$

The Lie derivative of the function $V: \mathbb{R}^{n} \rightarrow \mathbb{R}$ in the line of the smooth vector field $f: \mathbb{R}^{n} \rightarrow \mathbb{R}^{n}$ is calculated as follows:

$$
L_{f} V(x)=\partial V(x) / \partial x f(x)
$$

the Lie derivatives of higher orders are determined recurrently:

$$
L_{f}^{k} V(x)=L_{f}\left(L_{f}^{k-1} V(x)\right) .
$$

The following notation is used for convenience:

$$
\begin{aligned}
L_{f} h(x) & =\partial h(x) / \partial x f(x), \\
L_{G} h(x) & =\partial h(x) / \partial x G(x),
\end{aligned}
$$

here $G(x)=\operatorname{col}\left[g_{1}(x), \ldots, g_{m}(x)\right], g_{i}: \mathbb{R}^{n} \rightarrow \mathbb{R}^{n}$ are smooth vector functions, $i=\overline{1, m}$.

\subsection{Comparison Functions}

The continuous function $\sigma: \mathbb{R}_{+} \rightarrow \mathbb{R}_{+}$belongs to the class $\mathcal{K}$ if it is strictly increasing and $\sigma(0)=0$; it belongs to the class $\mathcal{K}_{\infty}$ if additionally it is proper unbounded; the continuous function $\beta: \mathbb{R}_{+} \times \mathbb{R}_{+} \rightarrow \mathbb{R}_{+}$from the class $\mathcal{K} \mathcal{L}$ if it belongs to the class $\mathcal{K}$ in the first argument for any fixed value of the second argument and strictly vanishes for the increasing second argument under any fixed value of the first argument. Such comparison functions were proposed in [71] (see also Section 5 in [53]). There are many methods of comparison for studying the dynamic systems (see [72-74]), the following lemma seems useful in the context of the present paper.

Lemma 6.1 [8]. For any continuous and positive definite function $\alpha: \mathbb{R}_{+} \rightarrow \mathbb{R}_{+}$, there exists the function $\beta_{\alpha} \in \mathcal{K} \mathcal{L}$ having the following property: if $y: \mathbb{R}_{+} \rightarrow \mathbb{R}_{+}$is any (locally) absolutely continuous function satisfying the differential inequality

$$
\dot{y}(t) \leqslant-\alpha(y(t)),
$$

almost for all $t \in \mathbb{R}_{+}$, then

$$
y(t) \leqslant \beta_{\alpha}(y(0), t)
$$

is satisfied for all $t \in \mathbb{R}_{+}$. 
Lemma 6.2 [23]. For any continuous and positive definite function $\alpha: \mathbb{R}_{+} \rightarrow \mathbb{R}_{+}$there exists a function $\beta_{\alpha} \in \mathcal{K} \mathcal{L}$ having the following property: if $y: \mathbb{R}_{+} \rightarrow \mathbb{R}_{+}$and $v: \mathbb{R}_{+} \rightarrow \mathbb{R}_{+}$are locally absolutely continuous and continuous functions satisfying the differential inequality

$$
\dot{y}(t) \leqslant-\alpha(\max \{y(t)+v(t), 0\})
$$

almost for all $t \in \mathbb{R}_{+}$, then

$$
y(t) \leqslant \max \left\{\beta_{\alpha}(y(0), t),\|v\|_{[0, t)}\right\}
$$

is satisfied for all $t \in \mathbb{R}_{+}$. If the inequality

$$
\dot{y}(t) \leqslant-\alpha(y(t))+v(t)
$$

is satisfied almost for all $t \in \mathbb{R}_{+}$, then the estimate

$$
y(t) \leqslant \beta_{\alpha}(y(0), t)+\int_{0}^{t} 2 v(s) d s
$$

is true for all $t \in \mathbb{R}_{+}$.

As was shown in [6], for any function $\beta \in \mathcal{K} \mathcal{L}$ there are functions $\chi, \sigma \in \mathcal{K}_{\infty}$ such that $\beta(s, t) \leqslant$ $\chi(s) \sigma\left(e^{-t}\right)$ for all $s, t \in \mathbb{R}_{+}$. The Lemmas 6.1 and 6.2 prove useful for stability analysis of the nonlinear dynamic systems with the use of the method of Lyapunov functions.

\subsection{Solutions of Dynamic Systems}

Let us consider the nonlinear system

$$
\dot{x}=f(x, u), \quad y=h(x),
$$

where $x \in \mathbb{R}^{n}, u \in \mathbb{R}^{m}, y \in \mathbb{R}^{p}$ are the vectors of state, input, and output, respectively; $f$ is a continuous and locally Lipschitzian vector function uniformly in $u$, that is, for any compact subsets $X \subset \mathbb{R}^{n}$ and $\Omega \subset \mathbb{R}^{m}$ there exists a constant $L>0$ such that $\left|f(x, u)-f\left(x^{\prime}, u\right)\right| \leqslant L\left|x-x^{\prime}\right|$ for all $x, x^{\prime} \in X$ and $u \in \Omega$; and $h$ is a continuous vector function, $h(0)=0, f(0,0)=0$. For the initial conditions $x_{0} \in \mathbb{R}^{n}$ and input $u \in M_{\mathbb{R}^{m}}$, let $x\left(t, x_{0}, u\right)$ be a unique maximal solution of system (1) (the notation $x(t)$ is used if the rest of the solution arguments are clear from the context; $\left.y\left(t, x_{0}, u\right)=h\left[x\left(t, x_{0}, u\right)\right]\right)$ defined over the finite interval $[0, T)$. The set $A$ is invariant for the system (6.1) with zero input if $T=+\infty$ and $x\left(t, x_{0}, 0\right) \in A, t \geqslant 0$ is satisfied for all $x_{0} \in A$. The set $A$ is uniformly invariant for system (6.1) with inputs $u \in M_{\Omega}$ if $T=+\infty$ and $x\left(t, x_{0}, u\right) \in A$, $t \geqslant 0$ are satisfied for all $x_{0} \in A$ and $u \in M_{\Omega}$. If $T=+\infty$ for all initial conditions $x_{0} \in \mathbb{R}^{n}$ and $u \in M_{\mathbb{R}^{m}}$, the system will be said to feature extendability of solutions. System (6.1) features unboundedness observability (UO) [75] if

$$
\limsup _{t \rightarrow T}\left|y\left(t, x_{0}, u\right)\right|=+\infty
$$

necessarily follows for each $x_{0} \in \mathbb{R}^{n}$ and input $u \in M_{\mathbb{R}^{m}}$ such that $T<+\infty$.

Stated differently, any unboundedness of the state vector can be observed using the output $y$. If the system output is bounded, then for the system the property of UO in this output means that the solutions are continuable. Any system has the property of $\mathrm{UO}$ in output $h(x)=x$. We stress that in the general case the UO property does not imply system solution extendability. The necessary and sufficient conditions for the properties of solution extendability and UO are presented in [75]. We give here some of them. 
Theorem 6.1. System (6.1) has solution extendability if and only if there exists a smooth and proper unbounded function $V: \mathbb{R}^{n} \rightarrow \mathbb{R}_{+}$satisfying the estimate

$$
D V(x) f(x, u) \leqslant V(x)+\sigma(|u|), \quad \sigma \in \mathcal{K}_{\infty}
$$

for all $x \in \mathbb{R}^{n}$ and $u \in \mathbb{R}^{m}$. If $u \in \Omega$, then Theorem 6.1 is valid if the inequality

$$
D V(x) f(x, u) \leqslant V(x)
$$

is satisfied for all $x \in \mathbb{R}^{n}$ and $u \in \Omega$. System (6.1) has the UO property if and only if there exists a smooth and proper unbounded function $V: \mathbb{R}^{n} \rightarrow \mathbb{R}_{+}$, corresponding for all $x \in \mathbb{R}^{n}$ and $u \in \mathbb{R}^{m}$ to the estimate

$$
D V(x) f(x, u) \leqslant V(x)+\sigma_{1}(|h(x)|)+\sigma_{2}(|u|), \quad \sigma_{1}, \sigma_{2} \in \mathcal{K}_{\infty} .
$$

Lemma 6.3. System (6.1) features solution extendability if and only if there exist functions $\chi_{1}$, $\chi_{2}$, and $\chi_{3}$ from the class $\mathcal{K}$ and $c \in \mathbb{R}_{+}$such that

$$
\left|x\left(t, x_{0}, u\right)\right| \leqslant \chi_{1}(t)+\chi_{2}\left(\left|x_{0}\right|\right)+\chi_{3}(|| u||)+c, \quad t \geqslant 0
$$

is satisfied for all $x_{0} \in \mathbb{R}^{n}$ and $u \in M_{\mathbb{R}^{m}}$. If $u \in \Omega$, then Lemma 6.3 retains its validity if the estimate

$$
\left|x\left(t, x_{0}, u\right)\right| \leqslant \chi_{1}(t)+\chi_{2}\left(\left|x_{0}\right|\right)+c, \quad t \geqslant 0
$$

is satisfied for all $x_{0} \in \mathbb{R}^{n}$ and $u \in M_{\Omega}$. System (6.1) has the UO property if and only if there exist functions $\chi_{1}, \chi_{2}, \chi_{3}$, and $\chi_{4}$ from the class $\mathcal{K}$ and $c \in \mathbb{R}_{+}$such that

$$
\left|x\left(t, x_{0}, u\right)\right| \leqslant \chi_{1}(t)+\chi_{2}\left(\left|x_{0}\right|\right)+\chi_{3}\left(\|u\|_{[0, t)}\right)+\chi_{3}\left(\|y\|_{[0, t)}\right)+c
$$

is satisfied for all $x_{0} \in \mathbb{R}^{n}, u \in M_{\mathbb{R}^{m}}$ and all $t \in[0, T)$.

System (6.1) is said to have the bounded-input-bounded-state (BIBS) property if the inequality

$$
\left|x\left(t, x_{0}, u\right)\right| \leqslant \max \left\{\vartheta\left(\left|x_{0}\right|\right), \vartheta(|| u||)\right\}, \quad t \geqslant 0,
$$

is satisfied for all $x_{0} \in \mathbb{R}^{n}$ and $u \in M_{\mathbb{R}^{m}}$ for some $\vartheta \in \mathcal{K}$. System (6.1) has the property of global stability modulo output (GSMO) if

$$
\left|x\left(t, x_{0}, u\right)\right| \geqslant \nu\left(\left|y\left(t, x_{0}, u\right)\right|\right), \quad t \in[0, \tilde{T}) \Rightarrow\left|x\left(t, x_{0}, u\right)\right| \leqslant \max \left\{\mu\left(\left|x_{0}\right|\right), \mu\left(\|u\|_{[0, \tilde{T})}\right)\right\}, \quad t \in[0, \tilde{T}),
$$

where the functions $\nu \in \mathcal{K}_{\infty}, \mu \in \mathcal{K}$ and $x_{0} \in \mathbb{R}^{n}, u \in M_{\mathbb{R}^{m}}, \tilde{T}<T$. If $u \in M_{\Omega}$, then in the last inequality the component $\mu\left(\|u\|_{[0, \tilde{T})}\right)$ can be omitted. Also if the set $Z=\{x: h(x)=0\}$ is compact, then system has the GSMO property.

Proposition 6.1. The following properties hold for system (6.1):

(1) $B I B S \Rightarrow G S M O \Rightarrow U O$;

(2) $B I B S \Rightarrow G S M O$ and output boundedness for all $x_{0} \in \mathbb{R}^{n}$ and $u \in M_{\mathbb{R}^{m}}$ :

$$
\left|y\left(t, x_{0}, u\right)\right| \leqslant \max \left\{\varsigma\left(\left|x_{0}\right|\right), \varsigma(\| u||)\right\}, \quad t \geqslant 0, \varsigma \in \mathcal{K} .
$$




\subsection{Lyapunov Stability Relative to Set}

The following material is based on the theoretical results formulated in [76] for system (6.1) with $u \in M_{\Omega}$. Let for system (6.1) given be an invariant (not necessarily compact) set $A$ satisfying the technical property met for the majority of applications:

$$
\sup _{x \in \mathbb{R}^{n}}\left\{|x|_{A}\right\}=+\infty .
$$

Definition 6.1. System (6.1) is called uniform global asymptotically stable (UGAS) relative to the set $A$ if its solutions are extendable and the following properties exist:

(1) Uniform stability. There is a function $\varphi \in \mathcal{K}_{\infty}$ such that

$$
\left|x\left(t, x_{0}, u\right)\right|_{A} \leqslant \varphi\left(\left|x_{0}\right|_{A}\right), \quad t \geqslant 0
$$

is satisfied for all $x_{0} \in \mathbb{R}^{n}$ and $u \in M_{\Omega}$.

(2) Uniform attractiveness. For any $\varepsilon, r>0$, there exists $T_{\varepsilon, r}>0$ such that

$$
\left|x\left(t, x_{0}, u\right)\right|_{A}<\varepsilon
$$

is true for each $u \in M_{\Omega}$ and $\left|x_{0}\right|_{A}<r$ for $t \geqslant T_{\varepsilon, r}$.

According to the following proposition, uniform stability and attractivity of system (6.1) are equivalent to the useful upper estimate of the system solution.

Proposition 6.2. System (6.1) is UGAS relative to the set $A$ if and only if it has the property of solution extendability and there exists a function $\beta \in \mathcal{K} \mathcal{L}$ such that

$$
\left|x\left(t, x_{0}, u\right)\right|_{A} \leqslant \beta\left(\left|x_{0}\right|_{A}, t\right), \quad t \geqslant 0
$$

is satisfied for all $x_{0} \in \mathbb{R}^{n}$ and $u \in M_{\Omega}$.

The UGAS formulation proposed in Proposition 6.2 as existence of a function from the class $\mathcal{K} \mathcal{L}$ is useful for practical purposes. For example, for the linear system (6.1) $\dot{x}=A x$ where the matrix $A$ has all eigennumbers with negative real part the set $A=\{0\}$ and $\beta\left(\left|x_{0}\right|, t\right)=a\left|x_{0}\right| e^{-b t}$ for some constants $a>0$ and $b>0$. In other words, functions of the class $\mathcal{K} \mathcal{L}$ enable one to generalize the exponential estimates used in the theory of linear systems to the solutions of nonlinear systems where the nature of decrease generally may be other than exponential.

Definition 6.2. The function $V: \mathbb{R}^{n} \rightarrow \mathbb{R}_{+}$which is smooth on $\mathbb{R}^{n} \backslash A$ is called the Lyapunov function relative to the set $A$ if it satisfies the following conditions:

(1) There exist $\alpha_{1}, \alpha_{2} \in \mathcal{K}_{\infty}$ such that for all $x \in \mathbb{R}^{n}$

$$
\alpha_{1}\left(|x|_{A}\right) \leqslant V(x) \leqslant \alpha_{2}\left(|x|_{A}\right)
$$

(2) There exists a continuous and positive definite function $\alpha_{3}: \mathbb{R}_{+} \rightarrow \mathbb{R}_{+}$such that

$$
D V(x) f(x, u) \leqslant-\alpha_{3}\left(|x|_{A}\right)
$$

for all $x \in \mathbb{R}^{n} \backslash A$ and $u \in \Omega$.

The property of smoothness of the function $V: \mathbb{R}^{n} \rightarrow \mathbb{R}_{+}$on $\mathbb{R}^{n} \backslash A$ should be understood as smoothness of the function $V$ for all $x \in \mathbb{R}^{n} \backslash A$, whereas $V(x)=0$ is satisfied for $x \in A$. The inequalities of item (1) of this definition mean for $A=\{0\}$ that the $V$ is a proper unbounded and positive definite function. 
Theorem 6.2. Let system (6.1) have the property of solution extendability. It is UGAS relative to the set $A$ if and only if there exists a smooth Lyapunov function relative to the set $A$. If $A$ is compact, then the requirement on solution extendability may be omitted.

For the case of $A=\{0\}, u \in M_{\{0\}}$, Theorem 6.2 coincides with the results of the classical Lyapunov theory [16] and extends this theory to the problem of uniform stability relative to set. There are other results developing the classical stability theory for equilibria to the stability in part of variables or output [57,77-79]. To illustrate the relation of Theorem 6.2 with this line of research in the stability theory of dynamic systems, we define $Z=\{x: h(x)=0\}$, a nonempty closed invariant set of system (6.1). Let there be some functions $\iota_{1}, \iota_{2} \in \mathcal{K}_{\infty}$ such that

$$
\iota_{1}\left(|x|_{Z}\right) \leqslant h(x) \leqslant \iota_{2}\left(|x|_{Z}\right)
$$

is satisfied for all $x \in \mathbb{R}^{n}$.

In what follows, we assume that such relation is always satisfied. It means that boundedness and tendency to zero of the output function give rise to a corresponding boundedness and tendency to zero of the distance of the trajectory representing point to the set $Z$ (and vice versa). Then, the property of stability relative to the set $Z$ in the sense of Definition 6.1 and the property of stability in output $y$ from [57] are equivalent. If the output function of a special form $h(x)=x_{1}$, where $x=\left[\begin{array}{ll}x_{1} & x_{2}\end{array}\right]$, was taken, then the relation between the presented results and the theory of stability in part of variables from $[77,79]$ becomes obvious. It is advisable to note that $[57,77,79]$ present sufficient conditions for various properties of stability not only in terms of existence in the system of a Lyapunov function from Definition 6.2, but also simplified stability criteria for some special cases. We cite one more useful property.

Lemma 6.4. System (6.1) is UGAS relative to the compact set $A$ for zero input $u$ if and only if there exist smooth semiproper unbounded function $W: \mathbb{R}^{n} \rightarrow \mathbb{R}_{+}$, function $\sigma \in \mathcal{K}$, and continuous positive definite function $\rho: \mathbb{R}_{+} \rightarrow \mathbb{R}_{+}$such that for all $x \in \mathbb{R}^{n}$ and $u \in \mathbb{R}^{m}$

$$
D W(x) f(x, u) \leqslant-\rho\left(|x|_{A}\right)+\sigma(|u|) .
$$

We recall that $W: \mathbb{R}^{n} \rightarrow \mathbb{R}_{+}$is called the semi-proper unbounded function if there exists a function $\pi \in \mathcal{K}$ and proper unbounded positive definite function $W_{0}: \mathbb{R}^{n} \rightarrow \mathbb{R}_{+}$such that $W=\pi \circ W_{0}$. The introduced complex of properties enables one to analyze for stability the nonlinear dynamic systems relative to the set uniformly in the unknown quasiconstant parameters and in the presence of external perturbations.

\subsection{Passivity of Dynamic System}

We note that there are different definitions of dissipativity of dynamic systems [80]. In the present work, by dissipativity is meant the following $[17,18,57,81]$.

Definition 6.3. System (6.1) is called dissipative with continuous function $V: \mathbb{R}^{n} \rightarrow \mathbb{R}_{+}$if the inequality

$$
V\left(x\left(x_{0}, u, t\right)\right) \leqslant V\left(x_{0}\right)+\int_{0}^{t} \varpi(x(\tau), y(\tau), u(\tau)) d \tau
$$

is true $\forall x_{0} \in \mathbb{R}^{n}, \quad u \in M_{\mathbb{R}^{m}}, t \geqslant 0$. The functions $\varpi$ and $V$ are called the expenditure function and supply rate, respectively.

For continuously differentiable supply rate, inequality (6.3) may be simplified as

$$
\dot{V}(x, u, t) \leqslant \varpi(x(t), u(t), y(t)) .
$$

The passive systems are the best known examples of the dissipative systems.

AUTOMATION AND REMOTE CONTROL Vol. 72 No. 82011 
Definition 6.4. System (6.1) is called passive with continuous function $V: \mathbb{R}^{n} \rightarrow \mathbb{R}_{+}$if for $\forall x_{0} \in \mathbb{R}^{n}, \quad u \in M_{\mathbb{R}^{m}}, \quad t \geqslant 0$ inequality (6.3) is true for the expenditure function $\varpi(x, y, u)=$ $y^{\mathrm{T}} u-\beta(x)$, where $\beta$ is a nonnegative continuous function. A system is called passive with a certain rate of dissipation $\beta$ if in (6.3) the equality sign can be used. A system is called passive without losses if in (6.3) it is possible to use the equality sign and $\beta(\cdot) \equiv 0$. System (6.1) is called strictly passive with continuous function $V: \mathbb{R}^{n} \rightarrow \mathbb{R}_{+}$if for $\forall x_{0} \in \mathbb{R}^{n}, u \in M_{\mathbb{R}^{m}}, t \geqslant 0$, inequality (6.3) is true for the expenditure function $\varpi(x, y, u)=y^{\mathrm{T}} u-\beta(x)$, where $\beta$ is a positive definite continuous system.

If $u(t) \equiv 0, t \geqslant 0$, and the supply rate is positive definite, then the passive system is Lyapunovstable and the strictly passive system is asymptotically stable. The Yakubovich-Kalman-Popov lemma determines a formula for the (strictly) passive system output

$$
\dot{x}=f(x)+G(x) u
$$

from the Lyapunov function $V$ (asymptotically) of the system stable under the zero input

$$
h(x)=L_{G} V(x) .
$$

The output function defines the set of zero dynamics $Z=\left\{x \in \mathbb{R}^{n}: h(x)=0\right\}$, the set of all system trajectories for identically zero output. On this set, the passive and strictly passive systems have stable and asymptotically stable dynamics.

\section{CONCLUSIONS}

In the present review, some fundamental results of the theory of input-state stable systems are compiled. This theory enables one to study robustness of the internal stability (state stability) of a system relative to the behavior of its output and input. The variety of the introduced properties and the profoundness of their consideration allowed the author of [3] to point to a new concept (or philosophy) of design and analysis of the nonlinear control systems. The publications devoted to the development and application of this concept allow one to agree with [3] and indicate to the indubitable importance of the results under consideration for the today theory of nonlinear control.

Unfortunately, the format of review does not allow us to consider the entire range of results obtained within the framework of this theory during the last two decades. For example, the results on extending the ISS property to the hybrid systems and systems with switchings [82-89] were left out of the scope of the present review. Among other interesting results that were not reviewed we note the following. The ISS property for system (2.3) was extended in [90] to the case where stability is influenced by the derivatives of the input vector, that is, where for any ( $k$-fold differentiable) input $u \in M_{\mathbb{R}^{m}}$ and $x_{0} \in \mathbb{R}^{n}$ there are functions $\beta \in \mathcal{K} \mathcal{L}, \gamma \in \mathcal{K}$ such that

$$
\left|x\left(t, x_{0}, u\right)\right| \leqslant \beta\left(\left|x_{0}\right|, t\right)+\sum_{i=0}^{k} \gamma\left(\left\|u^{(i)}\right\|\right)
$$

for $\forall t \geqslant 0$. We emphasize that in this case system (2.3) depends explicitly only on the vector $u$ but not on its derivatives. There exist systems that are not ISS but satisfy this property relative to the input and its derivatives [90]. Extension of the ISS property to the nonlinear systems with explicitly time dependent right-hand sides is given in [91], and the corresponding Lyapunov functions are discussed in [92] (see also [5]). Extension of the ISS property to the discrete systems like

$$
x_{t+1}=f\left(x_{t}, u_{t}\right), \quad x_{t} \in \mathbb{R}^{n}, \quad u_{t} \in \mathbb{R}^{m}, \quad f: \mathbb{R}^{n+m} \rightarrow \mathbb{R}^{n}
$$


where $t \geqslant 0$ is the discrete time, is presented in [93-95] (the estimate from Definition 2.1 retains its value for system (7.1)). The properties of a cascaded connection of systems of this type were discussed in [96]. The iISS property for system $(7.1) \alpha\left(\left|x\left(t, x_{0}, u\right)\right|\right) \leqslant \beta\left(\left|x_{0}\right|, t\right)+\sum_{i=0}^{t-1} \sigma\left(\left|u_{i}\right|\right)$ $\forall t \geqslant 0, x_{0} \in \mathbb{R}^{n}, u \in \mathbb{R}^{m}, \alpha, \sigma \in \mathcal{K}, \beta \in \mathcal{K} \mathcal{L}$ was shown in [97] to be equivalent to the property of global asymptotic stability of system (7.1). We stress that for the continuous systems (2.4) or (2.3) this statement is not true in the general case! In fact, additional conditions similar to those introduced in Lemma 5.1 are required in the continuous case. Probabilistic formulations of the ISS property for systems with stochastic noise were proposed in [98-100]. A concept of stability of autonomous nonlinear systems to almost all initial conditions which admits trajectories with the initial conditions belonging to the set of zero measure and not tending to the origin was considered in [101]. This property proves to be useful at studying systems with more than one equilibrium. Extension of the ISS property to this type of stability was proposed in [102]. Conditions for origination of input-state stability in averages and singularly perturbed systems were obtained in $[103,104]$. There are publications devoted to the problems of ISS stabilization of various canonical models of nonlinear systems [28,59] (see also [2]) that lie outside the scope of the present review.

\section{LIST OF ACRONYMS}

All terms introduced in the present paper and their acronyms are tabulated below.

$\begin{array}{lc}\text { input to state stability } & \text { ISS } \\ \text { integral input to state stability } & \text { ISS } \\ \text { input to output stability } & \text { IOS } \\ \text { 0-local stability } & \text { O-LS } \\ \text { asymptotic gain } & \text { AG } \\ \text { uniform asymptotic gain } & \text { UAG } \\ \text { limit property } & \text { LIM } \\ \text { input to state dynamical stability } & \text { ISDS } \\ \text { local input to state stability } & \text { LISS } \\ \text { output to state stability } & \text { OSS } \\ \text { input/output to state stability } & \text { IOSS } \\ \text { unboundedness observability } & \text { UO } \\ \text { output-Lagrange stable } & \text { OL } \\ \text { output-Lagrange input to output stable } & \text { OLIOS } \\ \text { state-independent input to output stable } & \text { SIIOS } \\ \text { robustly output stable } & \text { ROS } \\ \text { bounded input bounded state } & \text { BIBS } \\ \text { uniform global asymptotic stability } & \text { UGAS } \\ \text { control Lyapunov function } & \text { CLF } \\ \text { global stability modulo output } & \text { GSMO }\end{array}$

\section{ACKNOWLEDGMENTS}

This work was supported by Deutsche Forschungsgemeinschaft, project no. SFB 637.

\section{REFERENCES}

1. Kokotović, P. and Arcak, M., Constructive Nonlinear Control: A Historical Perspective, Automatica J. IFAC, 2001, no. 37(5), pp. 637-662. 
2. Sontag, E.D., Input to State Stability: Basic Concepts and Results, in Nonlinear and Optimal Control Theory, vol. 1932 of Lecture Notes in Math., Berlin: Springer, 2008, pp. 163-220.

3. Sontag, E.D., The ISS Philosophy as a Unifying Framework for Stability-like Behavior, in Nonlinear Control in the Year 2000, vol. 259 of Lecture Notes in Control and Inform. Sci., London: Springer, 2001, pp. 443-467.

4. Doyle, J., Francis, B., and Tannenbaum, A., Feedback Control Systems, New York: MacMillan, 1992.

5. Khalil, Kh.K., Nelineinye sistemy (Nonlinear Systems), Moscow: RKHD, 2009.

6. Sontag, E.D., Comments on Integral Variants of ISS, Syst. Control Lett., 1998, no. 34(1-2), pp. 93-100.

7. Natanson, I.P., Teoriya funktsii veshchestvennoi peremennoi (Theory of Functions of Real Variable), St. Petersburg: Lan', 1999.

8. Sontag, E.D., Smooth Stabilization Implies Coprime Factorization, IEEE Trans. Automat. Control, 1989, no. 34(4), pp. 435-443.

9. Dashkovskiy, S., Rüffer, B.S., and Wirth, F.R., Small Gain Theorems for Large Scale Systems and Construction of ISS Lyapunov Functions, 2009, accepted in SIAM J. Control and Optimization, available electronically: http://arxiv.org/pdf/0901.1842.

10. Sontag, E.D. and Wang, Y., New Characterizations of Input-to-State Stability, IEEE Trans. Automat. Control, 1996, no. 41(9), pp. 1283-1294.

11. Sontag, E.D., Further Facts about Input to State Stabilization, IEEE Trans. Automat. Control, 1990, no. 35(4), pp. 473-476.

12. Sontag, E.D. and Wang, Y., On Characterizations of the Input-to-State Stability Property, Syst. Control Lett., 1995, no. 24(5), pp. 351-359.

13. Teel, A.R., Connections between Razumikhin-type Theorems and the ISS Nonlinear Small Gain Theorem, IEEE Trans. Automat. Control, 1998, no. 43(7), pp. 960-964.

14. Karafyllis, I. and Jiang, Z.-P., A Small-gain Theorem for a Wide Class of Feedback Systems with Control Applications, SIAM J. Control Optim., 2007, no. 46(4), pp. 1483-1517.

15. Pepe, P., Karafyllis, I., and Jiang, Z.-P., On the Liapunov-Krasovskii Methodology for the ISS of Systems Described by Coupled Delay Differential and Difference Equations, Automatica J. IFAC, 2008, no. 44(9), pp. 2266-2273.

16. Lyapunov, A.M., Obshchaya zadacha ob ustoichivosti dvizheniya (General Problem of Motion Stability), Khar'kov: Khar'kov. Mat. Obshch., 1892.

17. Polushin, I.G., Fradkov, A.L., and Hill, D.J., Passivity and Passification of Nonlinear Systems, Autom. Remote Control, 2000, no. 3, pp. 355-388.

18. Willems, J.C., Dissipative Dynamical Systems. Part I: General Theory, Arch. Rational Mechanics Anal., 1972, no. 45, pp. 321-351.

19. Grüne, L., Input-to-State Dynamical Stability and Its Lyapunov Function Characterization, IEEE Trans. Automat. Control, 2002, no. 47(9), pp. 1499-1504.

20. Praly, L. and Wang, Y., Stabilization in Spite of Matched Unmodeled Dynamics and an Equivalent Definition of Input-to-State Stability, Math. Control Signals Syst., 1996, no. 9(1), pp. 1-33.

21. Sontag, E.D. and Teel, A., Changing Supply Functions in Input/State Stable Systems, IEEE Trans. Automat. Control, 1995, no. 40(8), pp. 1476-1478.

22. Malisoff, M., Rifford, L., and Sontag, E.D., Global Asymptotic Controllability Implies Input-to-State Stabilization, SIAM J. Control Optim., 2004, no. 42(6), pp. 2221-2238.

23. Angeli, D., Sontag, E.D., and Wang, Y., A Characterization of Integral Input-to-State Stability, IEEE Trans. Automat. Control, 2000, no. 45(6), pp. 1082-1097. 
24. Byrnes, C.I., Isidori, A., and Willems, J.C., Passivity, Feedback Equivalence, and the Global Stabilization of Minimum Phase Nonlinear Systems, IEEE Trans. Automat. Control, 1991, no. 36(11), pp. $1228-1240$.

25. Liberzon, D., Sontag, E.D., and Wang, Y., On Integral Input to State Stabilization, Proc. Am. Control Conf., San Diego, June 1999, pp. 1598-1602.

26. Angeli, D., Sontag, E.D., and Wang, Y., Further Equivalences and Semiglobal Versions of Integral Input to State Stability, Dynam. Control, 2000, no. 10(2), pp. 127-149.

27. Grüne, L., Asymptotic Behavior of Dynamical and Control Systems under Perturbation and Discretization, Lecture Notes Math., Berlin: Springer, 2002, vol. 1783.

28. Sepulchre, R., Janković, M., and Kokotović, P.V., Constructive Nonlinear Control, Berlin: Springer, 1997.

29. Sontag, E.D. and Wang, Y., Output-to-State Stability and Detectability of Nonlinear Systems, Syst. Control Lett., 1997, no. 29(5), pp. 279-290.

30. Polushin, I.G., On the Output Feedback Robust Stabilization of Passive Nonlinear Systems, Proc. 39th IEEE Conf. Decision Control, Sydney, December 2000, pp. 2934-2935.

31. Isidori, A., Nonlinear Control Systems, Berlin: Springer, 1995.

32. Liberzon, D., Morse, A.S., and Sontag, E.D., Output-Input Stability and Minimum-Phase Nonlinear Systems, IEEE Trans. Automat. Control, 2002, no. 47(3), pp. 422-436.

33. Sontag, E.D. and Wang, Y., A Notion of Input to Output Stability, Proc. Eur. Control Conf., Brussels, July 1997, CD-ROM file ECC958.pdf.

34. Hespanha, J.P., Liberzon, D., Angeli, D., and Sontag, E.D., Nonlinear Norm-observability Notions and Stability of Switched Systems, IEEE Trans. Automat. Control, 2005, no. 50(2), pp. 154-168.

35. Krichman, M., Sontag, E.D., and Wang, Y., Input-Output-to-State Stability, SIAM J. Control Optim., 2001, no. 39(6), pp. 1874-1928.

36. Sontag, E.D. and Wang, Y., Notions of Input to Output Stability, Syst. Control Lett., 1999, no. 38(4-5), pp. 235-248.

37. Sontag, E.D. and Wang, Y., Lyapunov Characterizations of Input to Output Stability, SIAM J. Control Optim., 2000, no. 39(1), pp. 226-249.

38. Ingalls, B. and Wang, Y., On Input-to-Output Stability for Systems not Uniformly Bounded, Proc. NOLCOS'01, St. Petersburg, July 2001.

39. Angeli, D., Ingalls, B., Sontag, E.D., and Wang, Y., Separation Principles for Input-Output and Integral-Input-to-State Stability, SIAM J. Control Optim., 2004, no. 43(1), pp. 256-276.

40. Lin, Y.D., Sontag, E.D., and Wang, Y., Input to State Stabilizability for Parametrized Families of Systems, Int. J. Robust Nonlinear Control, 1995, no. 5(3), pp. 187-205.

41. Sontag, E.D. and Wang, Y., Various Results Concerning Set Input-to-State Stability, Proc. 34th IEEE Conf. Decision and Control, New Orleans, December 1995, pp. 1330-1335,

42. Sontag, E.D. and Wang, Y., On Characterizations of Input-to-State Stability with Respect to Compact Sets, Proc. IFAC NOLCOS'95, Tahoe City, September 1995, pp. 226-231.

43. Jiang, Z.-P., Teel, A.R., and Praly, L., Small-gain Theorem for ISS Systems and Applications, Math. Control Signals Syst., 1994, no. 7(2), pp. 95-120.

44. Sontag, E.D. and Lin, Y., Stabilization with Respect to Noncompact Sets: Lyapunov Characterization and Effect of Bounded Inputs, Proc. NOLCOS'92, Bordeaux, 1992.

45. Efimov, D.V. and Fradkov, A.L., Input-to-Output Stabilization of Nonlinear Systems via Backstepping, Int. J. Robust Nonlinear Control, 2009, no. 19(6), pp. 613-633. 
46. Jiang, Z.-P., Mareels, I.M.Y., and Wang, Y., A Lyapunov Formulation of the Nonlinear Small-Gain Theorem for Interconnected ISS Systems, Automatica J. IFAC, 1996, no. 32(8), pp. 1211-1215.

47. Ito, H., State-dependent Scaling Problems and Stability of Interconnected iISS and ISS Systems, IEEE Trans. Automat. Control, 2006, no. 51(10), pp. 1626-1643.

48. Dashkovskiy, S., Rüffer, B.S., and Wirth, F.R., An ISS Small Gain Theorem for General Networks, Math. Control Signals Syst., 2007, no. 19(2), pp. 93-122.

49. Rüffer, B.S., Monotone Inequalities, Dynamical Systems, and Paths in the Positive Orthant of Euclidean $N$-space, Positivity, 2009, DOI:10.1007/s11117-009-0016-5.

50. Dashkovskiy, S., Rüffer, B.S., and Wirth, F.R., Applications of the General Lyapunov ISS Small-gain Theorem for Networks, Proc. 47th IEEE Conf. Decision Control, 2008, pp. 25-30.

51. Dashkovskiy, S. and Rüffer, B., Local ISS of Large-scale Interconnections and Estimates for Stability Regions, Syst. Control Lett., no. 59(3-4), pp. 241-247.

52. Arcak, M. and Teel, A., Input-to-State Stability for a Class of Lurie Systems, Automatica J. IFAC, 2002, no. 38(11), pp. 1945-1949.

53. Voronov, A.A., Ustoichivost', upravlyaemost', nablyudaemost' (Stability, Controllability, Observability), Moscow: Nauka, 1979.

54. Pervozvanskii, A.A., Kurs teorii avtomaticheskogo upravleniya. Uch. Posob. (Textbook on Automatic Control Theory), Moscow: Nauka, 1986.

55. Tsypkin, Ya.Z., Osnovy teorii avtomaticheskikh sistem (Fundamentals of the Theory of Automatic Systems), Moscow: Nauka, 1977. Translated under the title Grundlagen der Theorie automatischer Systeme, Berlin: VEB Verlag Technik, 1981.

56. Efimov, D.V., Robastnoe $i$ adaptivnoe upravlenie nelineinymi kolebaniyami (Robust and Adaptive Control of Nonlinear Oscillations), St. Petersburg: Nauka, 2005.

57. Miroshnik, I.V., Nikiforov, V.O., and Fradkov, A.L., Nelineinoe $i$ adaptivnoe upravlenie slozhnymi dinamicheskimi sistemami. Analiz $i$ sintez nelineinykh sistem (Nonlinear and Adaptive Control of Complex Dynamic Systems. Analysis and Design of Nonlinear Systems), St. Petersburg: Nauka, 2000.

58. Efimov, D.V., Passivity and Input-to-State Stability of Nonlinear Systems, Proc. 5th IFAC Sympos. on Robust Control Design, Toulouse, July 2006.

59. Krstic, M., Kanellakopoulos, I., and Kokotovic, P.V., Nonlinear and Adaptive Control Design, New York: Wiley, 1995.

60. Kolesnikov, A.A., Sinergeticheskaya teoriya upravleniya (Synergistic Control Theory), Moscow: Energoatomizdat, 1994.

61. Druzhinina, M.V., Nikiforov, V.O., and Fradkov, A.L., Adaptive Output Control Methods for Nonlinear Objects, Autom. Remote Control, 1996, no. 2, pp. 153-176.

62. Efimov, D.V. and Fradkov, A.L., Adaptive Input-to-Output Stabilization of Nonlinear Systems, Int. J. Adaptive Control Signal Process., 2008, no. 22(10), pp. 949-967.

63. Artstein, Z., Stabilization with Relaxed Controls, Nonlinear Anal., 1983, no. 7(11), pp. 1163-1173.

64. Sontag, E.D., A "Universal" Construction of Artstein's Theorem on Nonlinear Stabilization, Syst. Control Lett., 1989, no. 13(2), pp. 117-123.

65. Teel, A. and Praly, L., On Assigning the Derivative Of Disturbance Attenuation CLF, Proc. 37th IEEE CDC, Tampa, Florida, December 1998, pp 2497-2502.

66. Efimov, D.V., A Condition of CLF Existence for Affine Systems, Proc. 41st IEEE Conf. Decision Control, Las Vegas, 2002, pp. 1882-1887.

67. Efimov, D.V., Universal Formula for Output Asymptotic Stabilization, Proc. 15th IFAC World Congress 2002, T-We-M 07 4, Barselona, July 2002. 
68. Krstić, M. and Li, Z.-H., Inverse Optimal Design of Input-to-State Stabilizing Nonlinear Controllers, IEEE Trans. Automat. Control, 1998, no. 43(3), pp. 336-350.

69. Liberzon, D., Sontag, E.D., and Wang, Y., Universal Construction of Feedback Laws Achieving ISS and Integral-ISS Disturbance Attenuation, Syst. Control Lett., 2002, no. 46(2), pp. 111-127.

70. Alessandri, A., Observer Design for Nonlinear Systems by Using Input-to-State Stability, Proc. 43rd IEEE Conf. Decision Control, Bahamas, 2004, pp. 3892-3897.

71. Barbashin, E.A. and Krasovskii, N.N., On Existence of the Lyapunov Function in the Case of Asymptotic Stability as a Whole, Prikl. Mat. Mekh., 1954, vol. 18, pp. 345-350.

72. Chaplygin, S.A., Novyi metod priblizhennogo integrirovaniya differentsial'nykh uravnenii (A New Method of Approximate Integration of Differential Equations), Moscow: Gostekhizdat, 1948.

73. Lakshmikantham, V., Matrosov, V.M., and Sivasundaram, S., Vector Lyapunov Functions and Stability Analysis of Nonlinear Systems, vol. 63, Math. Appl., Dordrecht: Kluwer, 1991.

74. Leonov, G.A., Families of Transversal Curves for Two-dimensional Controls of Differential Equations, Vestn. S.-Peterburg. Gos. Univ., Ser. 1, 2006, vol. 4, pp. 48-78.

75. Angeli, D. and Sontag, E.D., Forward Completeness, Unboundedness Observability, and Their Lyapunov Characterizations, Syst. Control Lett., 1999, no. 38(4-5), pp. 209-217.

76. Lin, Y., Sontag, E.D., and Wang, Y., A Smooth Converse Lyapunov Theorem for Robust Stability, SIAM J. Control Optim., 1996, no. 34(1), pp. 124-160.

77. Vorotnikov, V.I. and Rumyantsev, V.V., Ustoichivost' $i$ upravlenie po chasti koordinat fazovogo vektora dinamicheskikh sistem: teoriya, metody i prilozheniya (Stability and Control in Part of Coordinates of the Vector of Dynamic Systems: Theory, Methods, and Applications), Moscow: Nauchnyi Mir, 2001.

78. Gelig, A.Kh., Leonov, G.A., and Yakubovich, V.A., Ustoichivost' nelineinykh sistem s needinstvennym polozheniem ravnovesiya (Stability of Nonlinear Systems with Nonunique Equilibrium), Moscow: Nauka, 1978.

79. Rumyantsev, V.V. and Oziraner, A.S., Ustoichivost' $i$ stabilizatsiya dvizhenii po otnosheniyu $k$ chasti peremennykh (Sability and Stabilization of Motions in Part of Variables), Moscow: Nauka, 1987.

80. Leonov, G.A., Burkin, I.M., and Shepeljavyi, A.I., Frequency Methods in Oscillation Theory, vol. 357, Math. Appl., Dordrecht: Kluwer, 1996. Translated from the 1992 Russian original and revised by the authors.

81. Hill, D.J. and Moylan, C.J., Dissipative Dynamical Systems: Basic Input-Output and State Properties, J. Franklin Inst., 1980, no. 309(5), pp. 327-357.

82. Cai, C. and Teel, A.R., Results on Input-to-State Stability for Hybrid Systems, Proc. 44th IEEE Conf. Decision Control, and the European Control Conference, Seville, 2005, pp. 5403-5408.

83. Cai, C. and Teel, A.R., Characterizations of Input-to-State Stability for Hybrid Systems, Syst. Control Lett., 2009, no. 58(1), pp. 47-53.

84. Brockett, R.W. and Liberzon, D., Quantized Feedback Stabilization of Linear Systems, IEEE Trans. Automat. Control, 2000, no. 45(7), pp. 1279-1289.

85. Hespanha, J.P., Liberzon, D., and Morse, A.S., Supervision of Integral-Input-to-State Stabilizing Controllers, Automatica J. IFAC, 2002, no. 38(8), pp. 1327-1335.

86. Liberzon, D., Switching in Systems and Control, in Systems 83 Control: Foundations 83 Applications, Boston: Birkhäuser Boston, 2003.

87. Mancilla-Aguilar, J.L. and García, R.A., On Converse Lyapunov Theorems for ISS and iISS Switched Nonlinear Systems, Syst. Control Lett., 2001, no. 42(1), pp. 47-53.

88. Vu, L., Chatterjee, D., and Liberzon, D., Input-to-State Stability of Switched Systems and Switching Adaptive Control, Automatica J. IFAC, 2007, no. 43(4), pp. 639-646. 
89. Xie, W., Wen, C., and Li, Z., Input-to-State Stabilization of Switched Nonlinear Systems, IEEE Trans. Automat. Control, 2001, no. 46(7), pp. 1111-1116.

90. Angeli, D., Sontag, E.D., and Wang, Y., Input-to-State Stability with Respect to Inputs and Their Derivatives, Int. J. Robust Nonlinear Control, 2003, no. 13(11), pp. 1035-1056.

91. Tsinias, J. and Karafyllis, I., ISS Property for Time-Varying Systems and Application to Partial-Static Feedback Stabilization and Asymptotic Tracking, IEEE Trans. Automat. Control, 1999, no. 44(11), pp. $2179-2184$.

92. Malisoff, M. and Mazenc, F., Further Remarks on Strict Input-to-State Stable Lyapunov Functions for Time-varying Systems, Automatica J. IFAC, 2005, no. 41(11), pp. 1973-1978.

93. Kazakos, D. and Tsinias, J., The Input to State Stability Condition and Global Stabilization of Discretetime Systems, IEEE Trans. Automat. Control, 1994, no. 39(10), pp. 2111-2113.

94. Jiang, Z.-P. and Wang, Y., Input-to-State Stability for Discrete-Time Nonlinear Systems, Automatica J. IFAC, 2001, no. 37(6), pp. 857-869.

95. Jiang, Z.-P. and Wang, Y., A Converse Lyapunov Theorem for Discrete-time Systems with Disturbances, Syst. Control Lett., 2002, no. 45(1), pp. 49-58.

96. Loria, A. and Nesic, D., Stability of Time-varying Discrete-time Cascades, Proc. 15th IFAC World Congress, Barcelona, June 2002.

97. Angeli, D., Intrinsic Robustness of Global Asymptotic Stability, Syst. Control Lett., 1999, no. 38(4-5), pp. 297-307.

98. Tsinias, J., Stochastic Input-to-State Stability and Applications to Global Feedback Stabilization, Int. J. Control, 1998, no. 71(5), pp. 907-930.

99. Deng, H. and Krstić, M., Output-feedback Stabilization of Stochastic Nonlinear Systems Driven by Noise of Unknown Covariance, Syst. Control Lett., 2000, no. 39(3), pp. 173-182.

100. Deng, H., Krstić, M., and Williams, R.J., Stabilization of Stochastic Nonlinear Systems Driven by Noise of Unknown Covariance, IEEE Trans. Automat. Control, 2001, no. 46(8), pp. 1237-1253.

101. Rantzer, A., A Dual to Lyapunov's Stability Theorem, Syst. Control Lett., 2001, no. 42(3), pp. 161-168.

102. Angeli, D., An Almost Global Notion of Input-to-State Stability, IEEE Trans. Automat. Control, 2004, no. $49(6)$, pp. $866-874$.

103. Christofides, P.D. and Teel, A.R., Singular Perturbations and Input-to-State Stability, IEEE Trans. Automat. Control, 1996, no. 41(11), pp. 1645-1650.

104. Nešić, D. and Teel, A.R., Input-to-State Stability for Nonlinear Time-varying Systems via Averaging, Math. Control Signals Syst., 2001, no. 14(3), pp. 257-280.

This paper was recommended for publication by B.T. Polyak, a member of the Editorial Board 